This item was submitted to Loughborough's Research Repository by the author.

Items in Figshare are protected by copyright, with all rights reserved, unless otherwise indicated.

\title{
Applications of Nijenhuis geometry II: maximal pencils of multihamiltonian structures of hydrodynamic type
}

PLEASE CITE THE PUBLISHED VERSION

https://doi.org/10.1088/1361-6544/abed39

\section{PUBLISHER}

IOP Publishing

\section{VERSION}

AM (Accepted Manuscript)

\section{PUBLISHER STATEMENT}

This is an Open Access Article. It is published by IOP under the Creative Commons Attribution 3.0 Unported Licence (CC BY). Full details of this licence are available at: http://creativecommons.org/licenses/by/3.0/

\section{LICENCE}

CC BY 3.0

\section{REPOSITORY RECORD}

Bolsinov, Alexey, Andrey Konyaev, and Vladimir Matveev. 2021. "Applications of Nijenhuis Geometry II: Maximal Pencils of Multihamiltonian Structures of Hydrodynamic Type”. Loughborough University. https://hdl.handle.net/2134/14214200.v1. 
PAPER • OPEN ACCESS

Applications of Nijenhuis geometry II: maximal pencils of multiHamiltonian structures of hydrodynamic type

To cite this article: Alexey V Bolsinov et al 2021 Nonlinearity 345136

View the article online for updates and enhancements. 


\title{
Applications of Nijenhuis geometry II: maximal pencils of multi-Hamiltonian structures of hydrodynamic type
}

\author{
Alexey V Bolsinov ${ }^{1, *}$, Andrey Yu Konyaev ${ }^{2}$ and \\ Vladimir S Matveev ${ }^{3}$ \\ 1 Department of Mathematical Sciences, Loughborough University, LE11 3TU, UK; \\ Faculty of Mechanics and Mathematics, Moscow State University and Moscow \\ Center for Fundamental and Applied Mathematics, 119992, Moscow, Russia \\ ${ }^{2}$ Faculty of Mechanics and Mathematics, Moscow State University, 119992, \\ Moscow, Russia \\ ${ }^{3}$ Institut für Mathematik, Friedrich Schiller Universität Jena, 07737 Jena, Germany \\ E-mail: A.Bolsinov@lboro.ac.uk, maodzund@yandex.ru and \\ vladimir.matveev@uni-jena.de
}

Received 28 September 2020, revised 20 February 2021

Accepted for publication 9 March 2021

Published 28 June 2021

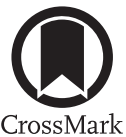

\begin{abstract}
We connect two a priori unrelated topics, the theory of geodesically equivalent metrics in differential geometry, and the theory of compatible infinitedimensional Poisson brackets of hydrodynamic type in mathematical physics. Namely, we prove that a pair of geodesically equivalent metrics such that one is flat produces a pair of such brackets. We construct Casimirs for these brackets and the corresponding commuting flows. There are two ways to produce a large family of compatible Poisson structures from a pair of geodesically equivalent metrics one of which is flat. One of these families is $(n+1)(n+2) / 2$ dimensional; we describe it completely and show that it is maximal. Another has dimension $\leqslant n+2$ and is, in a certain sense, polynomial. We show that a nontrivial polynomial family of compatible Poisson structures of dimension $n+2$ is unique and comes from a pair of geodesically equivalent metrics. In addition, we generalize a result of Sinjukov (1961) from constant curvature metrics to arbitrary Einstein metrics.
\end{abstract}

\footnotetext{
*Author to whom any correspondence should be addressed. Recommended by Professor Tamara Grava.
}

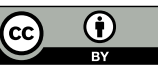

Original content from this work may be used under the terms of the Creative Commons Attribution 3.0 licence. Any further distribution of this work must maintain attribution to the author(s) and the title of the work, journal citation and DOI. 
Keywords: Nijenhuis operators, geodesically equivalent metrics, Poisson brackets of hydrodynamic type, multi-Hamiltonian structures, coupled $\mathrm{KdV}$ equations

Mathematics Subject Classification numbers: 37K05, 37K06, 37K10, 37K25, 37K50, 53B10, 53A20, 53B20, 53B30, 53B50, 53B99, 53D17.

\section{Introduction}

This paper continues the Nijenhuis geometry programme started in [3] and further developed in $[4,5,24]$. This programme was initially motivated by the fact that Niejnhuis operators (i.e. fields of endomorphisms $L=\left(L_{j}^{i}\right)$ with vanishing Nijenhuis torsion [31]) naturally appear in a number of different areas of geometry, algebra and mathematical physics. For this reason their normal forms, singularities and global properties deserve more systematic study than before. Although Nijenhuis operators usually serve as an auxiliary object in various mathematical constructions (two of them will be discussed in the present paper), in many cases their role is crucial and we intend to demonstrate this: the reader will notice that in many computations below we use $L$ as a primary object and this leads to essential simplifications and new results.

We believe that the appearance of Nijenhuis operators in various, seemingly unrelated research areas, might be an evidence of a hidden relationship between them. Indeed, one may often observe similarity of ideas, techniques or clever tricks used therein. Sometimes the relationship is much deeper and is manifested in 'overlapping' at the level of mathematical objects studied in these areas. In the present paper, we demonstrate such an overlap between geodesically equivalent pseudo-Riemannian metrics and compatible Poisson brackets of hydrodynamic type (see theorems 1-3 below). Once such a relationship is established and understood, one may try to transfer insights from one area to the other. That is what we do in theorem 5 by using our expertise in the theory of geodesically equivalent metrics to prove a uniqueness result for a certain type of Poisson pencils of hydrodynamic type.

Also we would like to emphasize that general methods of Nijenhuis geometry allows one to deal with singularities, i.e., those points where collisions of eigenvalues happen and $L$ changes its algebraic type. Traditionally, such singularities are excluded and, as a consequence, most constructions are local and restricted to a domain where $L$ reduces to some standard canonical form (for instance, is $\mathbb{R}$-diagonalizable with simple spectrum). In many problems, however, singularities cannot be ignored. First of all, this relates to global problems, when $L$ 'lives' on a closed manifold and singularities become unavoidable. In this paper, we either impose no additional restrictions on $L$, or assume that $L$ is differentially non-degenerate (see definition in [3, section 4.2] or just after theorem 5 below; this condition still allows singularities). Moreover, we give a description of all Nijenhuis operators geodesically compatible with a flat metric. Since this description is explicit, it can be used for analysis of singularities these operators may have.

\section{Basic definitions and main results}

We start with introducing two classes of objects we will be dealing with: Poisson structures of hydrodynamic type and geodesically equivalent metrics.

Given a metric $g$ of any signature on a manifold $M$ which is always assumed to be of dimension $n \geqslant 2$, for a function $h$, treated as the density of a Hamiltonian of hydrodynamic type, one can construct an operator $(=(1,1)$-tensor field $)$ by the formula 


$$
h \mapsto \nabla^{i} \nabla_{j} h=g^{i s} \frac{\partial^{2} h}{\partial x^{s} \partial x^{j}}-\Gamma_{j}^{i s} \frac{\partial h}{\partial x_{s}}
$$

(we use $g$ for index manipulations, for example $\Gamma_{j}^{i s}=\Gamma_{p j}^{s} g^{p i}$ ).

If the metric is flat, this construction has many parallels with the construction of the Hamiltonian vector field by a function $H$ and a Poisson bracket. Actually, a flat metric defines an infinite-dimensional Poisson bracket, the density $h$ defines a variational functional of hydrodynamic type and the operator $\nabla^{i} \nabla_{j} h$ determines the Hamiltonian flow generated by this functional. We refer to $[12,13,17,28]$ for details.

Parallels with and intuition coming from finite-dimensional Poisson brackets appeared to be very helpful in studying the following systems of partial differential equations: we view local coordinates $x^{1}, \ldots, x^{n}$ on $M^{n}$ as unknown functions which depend on two variables $t$ and $\tau$ and consider the following quasilinear systems of $n$ PDE on $n$ unknown functions of two coordinates:

$$
\frac{\partial}{\partial t} x^{i}(t, \tau)=A_{j}^{i}(x) \frac{\partial}{\partial \tau} x^{j}(t, \tau), \quad \text { where } A_{j}^{i}=\nabla^{i} \nabla_{j} h
$$

Such systems are called systems of hydrodynamic type in literature.

This construction was generalized in [21,22] for metrics of constant curvature $K$ : in this case the analog of formula (1) is

$$
h \mapsto \nabla^{i} \nabla_{j} h+K h \delta_{j}^{i}
$$

and correspondingly $A$ in (2) is given by $A_{j}^{i}(x)=\nabla^{i} \nabla_{j} h+K h \delta_{j}^{i}$.

Recall that two Poisson structures are compatible, if their sum is also a Poisson structure. In our infinite-dimensional situation, compatibility of two Poisson structures coming from constant curvature metrics $g$ and $\bar{g}$ is equivalent to the following two conditions (see e.g. the survey [30]):

(a) the operator $L_{j}^{i}:=\bar{g}^{i s} g_{s j}$ is a Nijenhuis operator, i.e., its Nijenhuis torsion vanishes, that is,

$$
\mathcal{N}_{L}(u, v)=L^{2}[u, v]-L[L u, v]-L[u, L v]+[L u, L v]=0
$$

for arbitrary vector fields $u$ and $v$ [31].

(b) For any $\alpha, \beta \in \mathbb{R}$ such that the operator $\alpha \mathrm{Id}+\beta L$ is invertible, the metric $\hat{g}=$ $g(\alpha \mathrm{Id}+\beta L)^{-1}$ has constant curvature $\hat{K}=\alpha K+\beta \bar{K}$ where $K$ and $\bar{K}$ are the curvatures of $g$ and $\bar{g}$ respectively.

Notice that by $[19,29]$ condition (a) is equivalent to the following property: for any $\alpha, \beta \in \mathbb{R}$ such that $\alpha \mathrm{Id}+\beta L$ is non-degenerate, the Christoffel symbols of the metric $\hat{g}=$ $g(\alpha \mathrm{Id}+\beta L)^{-1}$ are given by $\hat{\Gamma}_{k}^{i j}=\alpha \Gamma_{k}^{i j}+\beta \bar{\Gamma}_{k}^{i j}$. In the last formula, we raise the index $i$ of the Christoffel coefficients $\Gamma$ (resp. $\bar{\Gamma}, \hat{\Gamma}$ ) of the metric $g$ (resp. $\bar{g}, \hat{g}$ ) by its own metric.

We will call a set of constant curvature metrics Poisson-compatible, if any two metrics $g$ and $\bar{g}$ from this set satisfy (a) and (b).

Similar to the finite-dimensional case, the existence of a nontrivial compatible Poisson structure provides additional tools to analyse the system (2), in particular to construct explicit solutions and to study long-time behaviour of solutions. We refer to [22, 30, 36] for details.

Nijenhuis operators appeared naturally in the theory of geodesically equivalent metrics. Recall that two metrics $g$ and $\tilde{g}$ on one manifold are geodesically equivalent, if they have the same geodesics considered as Unparameterized curves. Let us consider the $(1,1)$-tensor 
$L=L(g, \tilde{g})$ defined by

$$
L_{j}^{i}:=\left(\frac{|\operatorname{det}(\tilde{g})|}{|\operatorname{det}(g)|}\right)^{\frac{1}{n+1}} \tilde{g}^{i k} g_{k j},
$$

where $\tilde{g}^{i j}$ is the (automatically, symmetric) tensor dual to $\tilde{g}_{i j}$ (in the sense $\tilde{g}^{i s} \tilde{g}_{j s}=\delta_{j}^{i}$ ). It is known [7] that for geodesically equivalent metrics the operator $L$ is Nijenhuis. Notice that $\tilde{g}$ can be recovered from $g$ and $L$ as

$$
\tilde{g}=\frac{1}{|\operatorname{det} L|} g L^{-1}
$$

which is equivalent to (4).

For a given $g$, we will call an operator $L$ geodesically compatible with $g$, if (5) defines a metric $\tilde{g}$ geodesically equivalent to $g$.

This condition is equivalent to the property that $L$ is self-adjoint with respect to $g$, nondegenerate and satisfies the system of PDEs (12) below. Sometimes speaking about geodesic compatibility of $L$ with $g$ one allows $L$ to be degenerate and only requires that $L$ is selfadjoint and satisfies (12). Actually since $\mathrm{Id}=\delta_{j}^{i}$ is geodesically compatible with every $g$ and equation (12) is linear, addition of const. · Id to $L$ makes it locally non-degenerate. In order to avoid misunderstanding, we will always either explicitly require the operator $L$ to be non-degenerate or allow it to be degenerate.

Our first result is the following relation between geodesically equivalent and Poissoncompatible metrics:

Theorem 1. Let $g$ be a flat metric. Then the following statements hold:

(a) If $L$ is geodesically compatible with $g$ and non-degenerate, then the metric $g L^{-1}$ has constant (possibly zero) curvature and is Poisson-compatible with $g$.

(b) If non-degenerate $L_{1}$ and $L_{2}$ are geodesically compatible with $g$, then the metrics $g L_{1}^{-1}$ and $\mathrm{gL}_{2}^{-1}$ are Poisson-compatible.

The first statement of this theorem means that the metrics $g$ and $\bar{g}=g L^{-1}$ define a family (pencil) of compatible Poisson brackets of hydrodynamic type, which can be used to construct integrable systems of quasilinear PDEs. One of such constructions is based on Casimir functions of the relevant Poisson structures. In the context of this paper, by a Casimir of the Poisson structure of hydrodynamic type (related to a metric $g$ with constant curvature $K$, see remark 7.1 for the definition of $K$ ) we understand a function $h: M \rightarrow \mathbb{R}$ which, if plugged into (3), produces the zero operator:

$$
\nabla^{i} \nabla_{j} h+K h \delta_{j}^{i}=0
$$

Since the property of $h$ to be a Casimir is completely defined in terms of $g$, for the sake of brevity, we will refer to it as a Casimir of $g$. If $K=0$ and $x^{1}, \ldots, x^{n}$ are flat coordinates for $g$, then the Casimirs are just linear combinations of the form $a_{0}+a_{1} x^{1}+\cdots+a_{n} x^{n}, a_{i} \in \mathbb{R}$. If $K \neq 0$, the Casimirs still form a vector space of dimension $n+1$ that admits a simple explicit description as soon as $g$ is given by means of a certain canonical model. For instance, if $g$ is the standard metric on the round sphere $S^{n} \subset \mathbb{R}^{n+1}$, then the Casimirs of $g$ are just restrictions of linear functions from $\mathbb{R}^{n+1}$ to $S^{n}$.

Let $g$ and $\bar{g}$ be two Poisson-compatible metrics and $h_{\alpha}$ be a Casimir of an arbitrary metric $g_{\alpha}=\left(g^{-1}-\alpha \bar{g}^{-1}\right)^{-1}, \alpha \in \mathbb{R}$ from the corresponding pencil. It is a well known fact that the Hamiltonian flows generated by $h_{\alpha}$ 's w.r.t. the Poisson bracket related to $g$ commute and this 
property leads to their integrability. However, if $h_{\alpha}$ is a common Casimir of $g_{\alpha}$ and $g$, then the corresponding flow vanishes and the above construction becomes trivial. For this reason it makes sense to distinguish essential Casimirs $h_{\alpha}$. The next theorem describes Casimirs and the corresponding commuting Hamiltonian flows for the pencil of Poisson-compatible metrics from theorem 1 .

Theorem 2. Let $g$ be a flat metric and $L$ be a non-degenerate geodesically compatible operator. Then, the following statements hold:

(a) The metrics $g$ and $\bar{g}=g L^{-1}$ have $n$ common independent Casimirs.

(b) For any $\alpha \in \mathbb{R}$ such that $\operatorname{det}(-\alpha L+\mathrm{Id})>0$, the function $h_{\alpha}=\sqrt{\operatorname{det}(-\alpha L+\mathrm{Id})}$ is a Casimir of the metric $g(-\alpha L+\mathrm{Id})^{-1}$.

(c) The Hamiltonian flow generated by $h_{\alpha}$ w.r.t. the Poisson bracket related to $g$ is given (up to a factor depending on $\alpha$ ) by the operator

$$
A_{\alpha}=\frac{1}{\sqrt{\operatorname{det}(-\alpha L+\mathrm{Id})}}(-\alpha L+\mathrm{Id})^{-1} .
$$

The flows given by $A_{\alpha}$ and $A_{\beta}$ commute for all $\alpha, \beta \in \mathbb{R}$.

Remark 2.1. Instead of commuting flows given by (6) and parameterized by $\alpha \in \mathbb{R}$, one usually considers the coefficients of the expansion of (6) in powers of $\alpha$ :

$$
\frac{1}{\sqrt{\operatorname{det}(-\alpha L+\mathrm{Id})}}(-\alpha L+\mathrm{Id})^{-1}=\mathrm{Id}+\alpha A_{1}+\alpha^{2} A_{2}+\alpha_{3} A_{3}+\cdots,
$$

with

$$
\begin{aligned}
& A_{1}=L+\frac{1}{2} \sigma_{1} \mathrm{Id} \\
& A_{2}=L^{2}+\frac{1}{2} \sigma_{1} L+\left(\frac{1}{2} \sigma_{2}+\frac{3}{8} \sigma_{1}\right) \mathrm{Id}, \\
& A_{3}=L^{3}+\frac{1}{2} \sigma_{1} L^{2}+\left(\frac{1}{2} \sigma_{2}+\frac{3}{8} \sigma_{1}\right) L+\left(\frac{1}{2} \sigma_{3}+\frac{3}{4} \sigma_{1} \sigma_{2}+\frac{5}{16} \sigma_{1}^{3}\right) \mathrm{Id},
\end{aligned}
$$

where $\sigma_{k}$ 's denote the coefficients of the characteristic polynomial $\operatorname{det}(\mathrm{Id}-\alpha L)=$ $1-\sigma_{1} \alpha-\cdots-\sigma_{n} \alpha^{n}$. The flows generated by these operators commute and hence define an integrable system of PDEs. They admit an infinite series of conservation laws given by the Casimir functions $h_{\alpha}=\sqrt{\operatorname{det}(-\alpha L+\text { Id })}$ or, equivalently, by the coefficients of the $\alpha$-power expansion $h_{\alpha}=1+\alpha F_{1}+\alpha^{2} F_{2}+\ldots$ :

$$
F_{1}=-\frac{1}{2} \sigma_{1}, \quad F_{2}=-\frac{1}{2} \sigma_{2}-\frac{1}{8} \sigma_{1}^{2}, \quad F_{3}=-\frac{1}{2} \sigma_{3}-\frac{1}{4} \sigma_{1} \sigma_{2}-\frac{1}{16} \sigma_{1}^{3}, \ldots
$$

Notice that the commuting flows and their conservation laws are defined in terms of a Nijenhuis operator $L$ only, whereas the metric $g$ in not involved in the final conclusion.

Integrability of the flow generated by $A_{1}$ that can be interpreted as a quasiclassical (dispersionless) limit of coupled KdV equations [1], was studied in detail by Ferapontov and Pavlov in [20]. This system is an example of the so-called integrable $\varepsilon$-systems introduced and studied by Pavlov in [32] for $\mathbb{R}$-diagonalizable operators $L$. These systems, in turn, are a particular case of a nice construction developed by Lorenzoni and Magri in [26] and based exclusively on a 
Nijenhuis operator $L$. No other ingredient is needed so that the system itself and its integrability can naturally be understood in the framework of Nijenhuis Geometry.

If we allow $L$ to be degenerate, then the operators geodesically compatible with $g$ form a vector space $\mathcal{L}_{g}$. Since all these operators $L$ are Nijenhuis, we may refer to $\mathcal{L}_{g}$ as a Nijenhuis pencil (we allow pencils of arbitrary dimension). According to the second statement of theorem 1 the pencil $\mathcal{L}_{g}$, in the case of a flat metric $g$, automatically leads to a large family of Poissoncompatible constant curvature metrics of the form $g L^{-1}$ (it is more appropriate here to consider contravariant metrics; they are given by the matrices $L g^{-1}=L^{i j}$ ). The following theorem provides an explicit description for them.

Theorem 3. Let $g$ be a flat metric and $x^{1}, \ldots, x^{n}$ be local coordinates in which all the components of $g$ are constant (i.e., local flat coordinates).

Then every operator $L$ geodesically compatible to $g$ with the index raised by $g$ is given by the following formula:

$$
L^{i j}=a^{i j}+b^{i} x^{j}+b^{j} x^{i}-K x^{i} x^{j} .
$$

Here $\left(a^{i j}, b^{i}, K\right)$ are constants and $a^{i j}=a^{j i}$. Conversely, every L given by (7) is geodesically compatible to $g$ and the components of $\frac{1}{2} \operatorname{grad}_{g}(\operatorname{tr} L)$ are $\lambda^{i}=b^{i}-K x^{i}$.

Moreover, near those points where $L^{i j}$ is non-degenerate and, therefore, defines a pseudoRiemannian contravariant metric, the curvature of this metric is constant and equals $K$.

Following [29, 30], we call a set of metrics compatible, if for any two of them, say $g$ and $\bar{g}$, the operator $L_{j}^{i}:=\bar{g}^{i s} g_{s j}$ is Nijenhuis (i.e., condition (a) from the definition of Poisson compatibility is fulfilled) and in addition for any $\alpha, \beta \in \mathbb{R}$ such that the operator $\alpha \mathrm{Id}+\beta L$ is non-degenerate, the curvature tensors of $g, \bar{g}$ and $\hat{g}=g(\alpha \mathrm{Id}+\beta L)^{-1}$ satisfy the relation

$$
\hat{R}_{k \ell}^{i j}=\alpha R_{k \ell}^{i j}+\beta \bar{R}_{k \ell}^{i j} \text {. }
$$

(In each curvature tensor we raised the index by its own metric).

Comparing this definition with the definition of Poisson-compatibility we see that Poisson compatible metrics are precisely compatible metrics of constant curvature.

Corollary 2.1. Let $g=\left(g_{i j}\right)$ be a flat metric on $\mathbb{R}^{n}$ and L an operator geodesically compatible with $g$. Then for any polynomials $P$ and $Q$, the metrics $g P(L)^{-1}$ and $g Q(L)^{-1}$ are compatible (whenever the operators $P(L)$ and $Q(L)$ are invertible). In particular, if the metrics $g, g L^{-1}, \ldots, g L^{-k}$ are of constant curvature (resp. flat), then $\left\{P(L) g^{-1}, \operatorname{deg} P \leqslant k\right\}$ is a pencil of Poisson-compatible (resp. flat) contravariant metrics.

Notice that every set $\left\{g_{0}, g_{1}, g_{2}, g_{3}, \ldots\right\}$ of Poisson-compatible metrics (or equivalently the corresponding pencil of compatible Poisson structures of hydrodynamic type) leads to a natural Nijenhuis pencil which is the linear span of all Nijenhuis operators of the form $L_{k}=g_{k}^{-1} g_{0}$, $k=0,1, \ldots$ In a very similar way, Nijenhuis pencils appear in the area of geodesically equivalent metrics and finite-dimensional multi-Hamiltonian structures and already for this reason they deserve to be studied as a separate subject in geometry. For instance, it would be interesting to describe maximal Nijenhuis pencils, i.e., those which are not contained in any larger Nijenhuis pencil. Notice that maximality of a Nijenhuis pencil would immediately imply maximality of the corresponding Poisson pencils and families of geodesically equivalent metrics (the converse, as a rule, is not true).

In this context, the pencil from theorem 3 admits the following interpretation. Consider an Euclidean metric $g \simeq \sum \mathrm{d}\left(x^{i}\right)^{2}$ and try to construct a big family of Poisson-compatible metrics 
containing $g$. Let us start with the trivial family of metrics whose components are constant in coordinates $x^{1}, \ldots, x^{n}$. The corresponding Nijenhuis pencil consists of symmetric operators with constant entries. Can this pencil be extended? And if yes, then how? The answer is that such an extension exists, is unique in the class of $g$-symmetric operators and coincides with the pencil from theorem 3 (corollary 5.1). In particular, the family of Poisson compatible metrics from theorem 3 is maximal (corollary 5.2).

Theorem 3 combined with theorem 1 gives us many examples of Poisson-compatible metrics and, consequently, integrable systems of hydrodynamic type. We discuss these examples in section 6. Some of these examples has features which were not observed in integrable systems of hydrodynamic type before. In particular, we construct nontrivial examples with Jordan blocks corresponding to nonconstant eigenvalues.

Corollary 2.1 suggests one more method for constructing multidimensional pencils of Poisson brackets of hydrodynamic type. Let us start with a flat metric $g$ and an operator $L$ geodesically compatible to it. We know that $g$ and $g L^{-1}$ are Poisson-compatible (and hence generate a pencil of dimension 2). By [34, theorem 1] (we reprove it in corollary 3.1) $g L^{-1}$ and $L$ are still geodesically compatible. Hence if $g L^{-1}$ is flat, we can repeat this procedure and consider the metric $g L^{-2}$ which will be Poisson-compatible with $g$ and $g L^{-1}$ by corollary 2.1 . If this metric is still flat, then we can make one more step and so on. If $g L^{-k}$ is flat but $g L^{-k-1}$ has non-zero constant curvature, then we show that the process stops as the next metric $g L^{-k-2}$ does not have constant curvature:

Theorem 4. Let L be geodesically compatible to an Einstein metric $g$ of constant non-zero scalar curvature and non-degenerate. If $\mathrm{d} \operatorname{tr}(L) \neq 0$, then the metric $g L^{-1}$ is not an Einstein metric of constant scalar curvature.

In theorem 4 we allow any dimension $n \geqslant 2$. In dimensions 2 and 3, Einstein metrics with constant scalar curvature are just metrics of constant curvature. In dimensions $\geqslant 3$ the scalar curvature of any Einstein metric is automatically constant.

In dimension $n \geqslant 3$, the analog of theorem 4 for metrics of constant curvature (which is in fact enough for our study of metrics $g L^{-k}$ ) is due to Sinjukov [34]. In dimension $n \geqslant 3$, our proof goes along the same lines as that by Sinjukov (which was merely sketched), but essentially uses results which were not available in the time of [34]. The proof in dimension $n=2$ is different, is partially based on the technology we develop in the present paper and as far as we know is new.

The example of a sequence $\left(g, g L^{-1}, \ldots, g L^{-k}\right.$ flat, $g L^{-k-1}$ of constant non-zero curvature) with $k=n$ was constructed in [20] and is as follows: the metric $g$ and operator $L$ are given in local coordinates $x^{1}, \ldots, x^{n}$ by

$$
g=\sum_{i=1}^{n} \prod_{j \neq i}\left(x^{i}-x^{j}\right)\left(\mathrm{d} x^{i}\right)^{2} \quad \text { and } \quad L=\operatorname{diag}\left(x^{1}, \ldots, x^{n}\right) .
$$

One can slightly generalize this example to include the points at which the operator $L$ is not diagonalizable. In the context of the present paper, this generalization is essentially due to [3], where we discussed singularities of geodesically compatible pairs: the metric is [3, equation (37)] and the operator is [3, equation (36)]. We repeat these formulas here: 


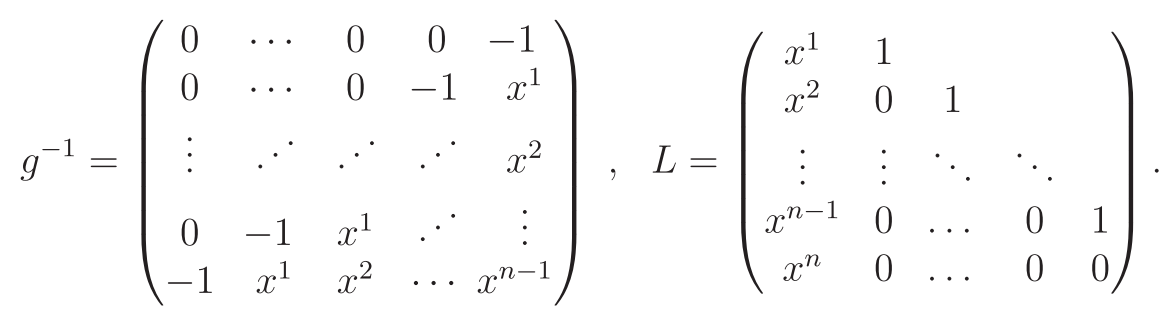

Much earlier, this form of $g$ and $L$ appeared in the study of multi-Hamiltonian structures for the coupled KdV system by Antonowicz and Fordy [1]. The dispersionless limit of the corresponding Poisson structures is essentially the same as the set of Poisson-compatible metrics generated by $g, g L^{-1}, \ldots, g L^{-n}$ with $g$ and $L$ given by (10).

Formulas (9) and (10) are related as follows: if one writes the pair (9) in the coordinates $\left(\sigma_{1}, \ldots, \sigma_{n}\right)$, where $\sigma$ 's are the coefficients of the characteristic polynomial $\operatorname{det}(t \mathrm{Id}-L)=$ $t^{n}-\sigma_{1} t^{n-1}-\sigma_{2} t^{n-2}-\cdots-\sigma_{n}$, one obtains (up to a sign) the pair (10). Of course, the inverse transformation, from (10) to (9), is possible only near those points where $L$ has $n$ different real-valued eigenvalues.

In both cases, the metrics $g, g L^{-1}, \ldots, g L^{-n}$ are flat and $g L^{-n-1}$ has constant non-zero curvature. All together they generate a pencil of compatible Poisson brackets of hydrodynamic type of dimension $n+2$. It is not hard to see that this pencil is maximal. Our next result shows that a pencil with such properties is unique.

Theorem 5. Let L be a Nijenhuis operator which is invertible, self-adjoint with respect to a metric $g$ and differentially non-degenerate almost everywhere. Suppose the metrics $g L^{-k}$ are flat for $k=0, \ldots, n$ and the metric $g L^{-n-1}$ has constant curvature $K \neq 0$. Then $g$ and $L$ are geodesically compatible. Moreover, in a neighborhood of every point, where L is differentially non-degenerate, the pair $(g, L)$ is locally isomorphic (up to multiplication of $g$ by a constant) to $(10)$.

Recall that according to [3, section 4.2] an operator $L$ is called differentially non-degenerate at a point if the differentials of the coefficients of its characteristic polynomial are linearly independent at this point.

Remark 2.2. For dimension two, theorem 5 follows from [22, theorem 10]. In arbitrary dimension, a similar statement was announced in [20], but to the best of our knowledge, the proof has never been published.

Remark 2.3. The case when also the metric $g L^{-n-1}$ is flat is much easier (provided $L$ is differentially non-degenerate and therefore is diagonalizable almost everywhere). We discuss it within the proof, in remark 7.3. In this case essentially only the following example and its natural modifications with complex-valued eigenvalues are possible: the metric is $g=\operatorname{diag}\left(g_{1}\left(x^{1}\right), \ldots, g_{n}\left(x^{n}\right)\right)$ and the operator is $L=\operatorname{diag}\left(\ell_{1}\left(x^{1}\right), \ldots, \ell_{n}\left(x^{n}\right)\right)$. In this case, generators of commuting flows coming from this multi-Hamiltonian structure are all functions of the Nijenhuis operator $L$ and are therefore Nijenhuis operators also. In this case, the metric $g$ is not geodesically compatible with $L$. Systems of hydrodynamic type (2) such that $A$ is a Nijunhuis operator are well-understood in the case when $L$ is diagonalizable; they decouple in Hopf equations and can be solved (almost) explicitly. An inclusion of singular points to this situation was done in [4].

Note that if we do not assume that $L$ is differentially non-degenerate, then there are nontrivial examples when the above explained process does not stop so that all the metrics 
of the form $g L^{-k}, k \in \mathbb{N}$ are flat and we obtain an infinite-dimensional Poisson pencils of hydrodynamic type (see example 6.1).

\section{Proof of theorem 1}

Let $L$ be an operator geodesically compatible with a metric $g$ of any signature. We do not assume a priori that the metric is flat. We consider the function $\lambda:=\frac{1}{2} \operatorname{tr} L$, and its differential $\mathrm{d} \lambda$ whose components will be denoted by $\lambda_{i}=\frac{\partial \lambda}{\partial x^{i}}$ and we denote the components of $\operatorname{grad}_{g} \lambda$ by $\lambda^{i}:=g^{\text {is }} \lambda_{s}$.

We start with Lemma describing the relationship between Christoffel symbols of $\nabla$ and $\bar{\nabla}$, the Levi-Civita connections of the metrics $g$ and $\bar{g}=g L^{-1}$.

Lemma 3.1. Let $g$ and $L$ be geodesically compatible and $L$ be non-degenerate. Then the Christoffel symbols of $\bar{g}=g L^{-1}$ are given by

$$
\bar{\Gamma}_{j k}^{i}=\Gamma_{j k}^{i}-\lambda^{i} \bar{g}_{j k}
$$

where $\Gamma_{j k}^{i}$ are the Christoffel symbols of $g$.

Proof. We use the equation of geodesic compatibility (see e.g. [7, theorem 2]):

$$
\nabla_{k} L_{i j}=\lambda_{i} g_{j k}+\lambda_{j} g_{i k}, \quad \text { with } \quad L_{i j}=L_{i}^{s} g_{s j},
$$

which we rewrite raising two indexes by $g$ as

$$
\nabla_{k} L^{i j}=\lambda^{i} \delta_{k}^{j}+\lambda^{j} \delta_{k}^{i}, \quad \text { with } \quad L^{i j}=L_{s}^{i} g^{s j} .
$$

Notice that $L^{i j}=\bar{g}^{i j}$ (where $\bar{g}^{i s} \bar{g}_{s j}=\delta_{j}^{i}$ as usual). Hence (13) gives

$$
\frac{\partial \bar{g}^{i j}}{\partial x^{k}}+\bar{g}^{i s} \Gamma_{s k}^{j}+\bar{g}^{j s} \Gamma_{s k}^{i}=\lambda^{i} \delta_{k}^{j}+\lambda^{j} \delta_{k}^{i}
$$

or, equivalently,

$$
\frac{\partial \bar{g}^{i j}}{\partial x^{k}}+\bar{g}^{i s}\left(\Gamma_{s k}^{j}-\lambda^{j} \bar{g}_{s k}\right)+\bar{g}^{j s}\left(\Gamma_{s k}^{i}-\lambda^{i} \bar{g}_{s k}\right)=0 .
$$

This means that $\bar{g}$ is parallel w.r.t. the symmetric connection whose Christoffel symbols $\bar{\Gamma}_{j k}^{i}$ are defined by (11). Thus, this is the Levi-Civita connection for $\bar{g}$, as stated.

Corollary 3.1 (Essentially, [34]). Let $g$ be geodesically compatible with a nondegenerate operator $L$. Then $\bar{g}=g L^{-1}$ is geodesically compatible with $L$.

Proof. Using the identity $\nabla g=0$ and lemma 3.1 we get

$$
\begin{aligned}
\bar{\nabla}_{k}\left(L_{i}^{s} \bar{g}_{s j}\right) & =\bar{\nabla}_{k} g_{i j}=\frac{\partial g_{i j}}{\partial x^{k}}-g_{j s} \bar{\Gamma}_{i k}^{s}-g_{j s} \bar{\Gamma}_{i k}^{s} \\
& =g_{i s}\left(\Gamma_{j k}^{s}-\bar{\Gamma}_{j k}^{s}\right)+g_{j s}\left(\Gamma_{i k}^{s}-\bar{\Gamma}_{i k}^{s}\right) \\
& =g_{i s} \lambda^{s} \bar{g}_{j k}+g_{j s} \lambda^{s} \bar{g}_{i k}=\lambda_{i} \bar{g}_{j k}+\lambda_{j} \bar{g}_{i k} .
\end{aligned}
$$

It remains to notice that $\bar{\nabla}_{k}\left(L_{i}^{s} \bar{g}_{s j}\right)=\lambda_{i} \bar{g}_{j k}+\lambda_{j} \bar{g}_{i k}$ is exactly the condition (12) for $\bar{g}$ and $L$. 
Lemma 3.2 ([34] in dimensions $\boldsymbol{n} \geqslant 3)$. Assume that $g$ and L are geodesically compatible, $g$ is flat and $L$ is non-degenerate. Then $\bar{g}=g L^{-1}$ has a constant, possibly zero, curvature.

Proof. For a metric $g$, we consider the following system of linear partial differential equations on the function $h$ :

$$
\nabla_{j} \nabla^{i} h-\frac{1}{n}\left(\nabla^{s} \nabla_{s} h\right) \delta_{j}^{i}=0
$$

This equation naturally appeared in different parts of differential geometry and is well understood; let us recall the following known property of this equation:

If the space of solutions of (14) is at least $n+1$-dimensional, then the metric has constant curvature (for $n \geqslant 3$, see [11, end of page 343]).

We did not find the case $n=2$ in the literature so let us give a proof. Consider the canonical complex structure $J=J_{j}^{i}$ (which is the $g$-volume form with one index raised) and observe that (14) implies that the 'skew gradient' vector $J_{s}^{i} \nabla^{s} h$ is Killing. Indeed, $\nabla_{j}\left(J_{\beta}^{i} \nabla^{\beta} h\right)=J_{\beta}^{i} \nabla_{j} \nabla^{\beta} h=c J_{j}^{i}$ is $g$-skew-symmetric (with $c=\frac{1}{n} \nabla_{s} \nabla^{s} h$ ) so that the Killing equation is fulfilled. The existence of three linearly independent solutions of (14) implies the existence of two linearly independent Killing vector fields so the curvature is constant, as claimed.

For each function $h$ in view of (11), its second $\bar{g}$-covariant derivative reads:

$$
\bar{\nabla}_{j} \bar{\nabla}_{i} h=\nabla_{j} \nabla_{i} h+\lambda^{s}\left(\nabla_{s} h\right) \bar{g}_{j i}
$$

Since $g$ is flat, locally it has $n+1$ linearly independent functions $h$ such that

$$
\nabla_{j} \nabla_{i} h=0
$$

In flat coordinates $y^{1}, \ldots, y^{n}$ for $g$ (i.e., such that $\Gamma_{j k}^{i}=0$ ), these functions take the form $h=\alpha_{0}+\alpha_{1} y^{1}+\cdots+\alpha_{n} y^{n}$. In view of (15) they also satisfy $\bar{\nabla}_{j} \bar{\nabla}_{i} h=\lambda^{s}\left(\nabla_{s} h\right) \bar{g}_{j i}$ which immediately implies (14) with respect to the metric $\bar{g}$. Thus, the dimension of the space of solutions of (14) for $\bar{g}$ is at least $n+1$ and hence $\bar{g}$ has constant curvature.

Lemma 3.2 proves the first part of the first statement of theorem 1. By construction, the operator $L$ is Nijenhuis (as an operator geodesically compatible with $g$ ), so the property (a) from the definition of Poisson-compatible metrics is automatically fulfilled. It remains to check property (b), we will do it at the end of this section.

Now let us discuss the second statement of theorem 1 . Since $L_{1}$ and $L_{2}$ are geodesically compatible with $g$, the metrics $g_{1}=g L_{1}^{-1}$ and $g_{2}=g L_{2}^{-1}$ have constant curvature by lemma 3.2. Recall that operators compatible with a fixed metric $g$ are all Nijenhuis and form a vector space (Nijenhuis pencil). In particular, $\alpha L_{1}+\beta L_{2}$ is a Nijenhuis operator for all $\alpha, \beta \in \mathbb{R}$. We, however, need to check this property for the operator $\left(g_{1}\right)^{-1} g_{2}=L_{1} L_{2}^{-1}$. To that end, we prove the following general fact from Nijenhuis geometry which is important on its own.

Lemma 3.3. Consider a pair of non-degenerate Nijenhuis operators $L_{1}$ and $L_{2}$. Then $\alpha L_{1}+\beta L_{2}$ is Nijenhuis operator for arbitrary constants $\alpha, \beta \in \mathbb{R}$ if and only if $L_{1} L_{2}^{-1}$ is Nijenhuis operator. 
Proof. It is well known that if $L_{1}$ and $L_{2}$ are both Nijenhuis, then $\alpha L_{1}+\beta L_{2}$ is Nijenhuis if and only if the Frolicher-Nijenhuis bracket of $L_{1}$ and $L_{2}$ vanishes (see [15] and e.g. [3]):

$$
\begin{aligned}
{\left[\left[L_{1}, L_{2}\right]\right]=} & L_{1}\left[L_{2} v, w\right]+L_{1}\left[v, L_{2} w\right]+L_{2}\left[L_{1} v, w\right]+L_{2}\left[v, L_{1} w\right] \\
& -L_{1} L_{2}[v, w]-L_{2} L_{1}[v, w]-\left[L_{1} v, L_{2} w\right]-\left[L_{2} v, L_{1} w\right]=0 .
\end{aligned}
$$

Here $v, w$ are arbitrary vector fields and [, ] stands for standard Lie bracket of vector fields. Let us prove the following identity, which immediately implies the statement of the lemma:

$$
\begin{aligned}
& \mathcal{N}_{L_{1}}(v, w)+L_{1} L_{2}^{-1} L_{1} L_{2}^{-1} \mathcal{N}_{L_{2}}(v, w)-\mathcal{N}_{L_{1} L_{2}^{-1}}\left(L_{2} v, L_{2} w\right) \\
& =L_{1} L_{2}^{-1}\left[\left[L_{1}, L_{2}\right]\right](v, w) .
\end{aligned}
$$

We have

$$
\begin{aligned}
& \mathcal{N}_{L_{1}}(v, w)+L_{1} L_{2}^{-1} L_{1} L_{2}^{-1} \mathcal{N}_{L_{2}}(v, w)-\mathcal{N}_{L_{1} L_{2}^{-1}}\left(L_{2} v, L_{2} w\right) \\
&=L_{1} L_{2}^{-1} L_{2}\left[L_{1} v, w\right]+L_{1} L_{2}^{-1} L_{2}\left[v, L_{1} w\right]-L_{1} L_{2}^{-1} L_{2} L_{1}[v, w] \\
& \quad-\left[L_{1} v, L_{1} w\right]+L_{1} L_{2}^{-1} L_{1}\left[L_{2} v, w\right]+L_{1} L_{2}^{-1} L_{1}\left[v, L_{2} w\right] \\
& \quad-L_{1} L_{2}^{-1} L_{1} L_{2}[v, w]-L_{1} L_{2}^{-1} L_{1} L_{2}^{-1}\left[L_{2} v, L_{2} w\right]-L_{1} L_{2}^{-1}\left[L_{1} v, L_{2} w\right] \\
& \\
& \quad-L_{1} L_{2}^{-1}\left[L_{2} v, L_{1} w\right]+L_{1} L_{2}^{-1} L_{1} L_{2}^{-1}\left[L_{2} v, L_{2} w\right]+\left[L_{1} v, L_{1} w\right] \\
&= L_{1} L_{2}^{-1}\left[\left[L_{1}, L_{2}\right]\right](v, w),
\end{aligned}
$$

as stated.

To complete the proof of theorem 1 (both items 1 and 2), it remains to show that the curvature of the metric $g L^{-1}$ linearly depends on $L$, where $L$ is now understood as an element for the vector space (Nijenhuis pencil) of all operators geodesically compatible with $g$. This will be implied by the following lemma:

Lemma 3.4. Let $g$ be a flat metric, L be a geodesically compatible non-degenerate operator and $K \in \mathbb{R}$ be the curvature of $g L^{-1}$.

Then,

$$
\nabla^{i} \nabla_{j} \lambda+K \delta_{j}^{i}=0
$$

Proof. This identity will be derived from the following algebraic relation between the curvature tensor $R$ of a metric $g$ and an operator $L$ geodesically compatible to it, see e.g. [6, equation (13) and theorem 7]:

$$
[R(v, u), L]=[u \otimes g(v)-v \otimes g(u), M], \quad \text { where } M_{j}^{i}=\nabla^{i} \nabla_{j} \lambda .
$$

Here $u$ and $v$ are arbitrary tangent vectors and [,] denotes the standard matrix commutator $[A, B]=A B-B A$.

W.l.o.g. we will assume that $L$ is not proportional to the identity (recall that if $L=f(x) \operatorname{Id}$, then $f(x)=$ const [38] and the statement becomes trivial). Since in our case $g$ is flat, then $R(v, u)=0$ implying $M=\rho$ Id (as $M$ commutes with any matrix of the form $u \otimes g(v)-v \otimes$ $g(u)$ ). To show that $\rho=-K$, we apply (18) once again for $\bar{g}=g L^{-1}$ and $L$ (as they are still geodesically compatible by corollary 3.1 ):

$$
[\bar{R}(v, u), L]=[u \otimes \bar{g}(v)-v \otimes \bar{g}(u), \bar{M}], \quad \bar{M}_{j}^{i}=\bar{\nabla}^{i} \bar{\nabla}_{j} \lambda .
$$


Since $\bar{g}$ is of constant curvature $K$, then $\bar{R}(v, u)=K(v \otimes \bar{g}(u)-u \otimes \bar{g}(v))$. On the other hand, formula (15) gives:

$$
\bar{\nabla}^{i} \bar{\nabla}_{j} \lambda=L_{s}^{i} \nabla^{s} \nabla_{j} \lambda+\lambda_{s} \lambda^{s} \delta_{j}^{i} \quad \text { or, in our case, } \quad \bar{M}=\rho L+\lambda^{s} \lambda_{s} \text { Id } .
$$

Hence, (19) can be rewritten in the form

$$
[v \otimes \bar{g}(u)-u \otimes \bar{g}(v),(K+\rho) L]=0 \quad \text { for all } u \text { and } v,
$$

implying $K+\rho=0$, that is $M_{j}^{i}=\nabla^{i} \nabla_{j} \lambda=-K \delta_{j}^{i}$, as required.

Now consider two operators $L_{1}$ and $L_{2}$ compatible with $g$ and apply lemma 3.4 for the linear combination $L=\alpha L_{1}+\beta L_{2}$. We denote the curvatures of $L, L_{1}$ and $L_{2}$ by $K, K_{1}, K_{2}$, and similarly $\lambda=\frac{1}{2} \operatorname{tr} L, \lambda_{1}=\frac{1}{2} \operatorname{tr} L_{1}, \lambda_{2}=\frac{1}{2} \operatorname{tr} L_{2}$. Then lemma 3.4 gives

$$
K \delta_{j}^{i}=\nabla^{i} \nabla_{j} \lambda=\nabla^{i} \nabla_{j}\left(\alpha \lambda_{1}+\beta \lambda_{2}\right)=\left(\alpha K_{1}+\beta K_{2}\right) \delta_{j}^{i}
$$

Hence, $K=\alpha K_{1}+\beta K_{2}$, as required. This completes the proof of the second statement of theorem 1. For the first statement, the end of proof is exactly the same. One just need to replace $L_{1}$ and $L_{2}$ with Id and $L$. Theorem 1 is proved.

\section{Proof of theorem 2}

We consider a flat metric $g$ and a non-degenerate operator $L$ geodesically compatible to it. From theorem 1 we already know that $\bar{g}=g L^{-1}$ is a metric of constant curvature $K$.

Recall that Casimir functions $h$ for $g$ are those satisfying the equation

$$
\nabla^{i} \nabla_{j} h=0
$$

whereas the equation for Casimirs of $\bar{g}=g L^{-1}$ is

$$
\bar{\nabla}^{i} \bar{\nabla}_{j} h+K h \delta_{j}^{i}=0 .
$$

In view of (15), the latter equation can also be rewritten in the form

$$
\bar{g}^{s i} \nabla_{s} \nabla_{j} h+\left(\lambda^{s} \nabla_{s} h+K h\right) \delta_{j}^{i}=0 .
$$

We first show that the metrics $g$ and $\bar{g}$ have 'many' common Casimirs. The next lemma is equivalent to the first statement of theorem 2 .

Lemma 4.1. The vector space of Casimirs of $\bar{g}$ contains a subspace of codimension one that consists of Casimirs of $g$.

Proof. Let $h$ be a Casimir of $\bar{g}=g L^{-1}$. Taking into account that $\bar{g}^{s i}=L^{s i}$, we can rewrite (22) in the form

$$
L^{s i} \nabla_{s} \nabla_{j} h=\rho \delta_{j}^{i} \quad \text { or, equivalently, } \quad \nabla_{s} \nabla_{j} h=\rho\left(L^{-1}\right)_{j}^{r} g_{r s}
$$

with $\rho=-\lambda^{s} \nabla_{s} h-K h$. Thinking of $\rho$ as an unknown function, we will derive some additional conditions for it.

Taking $\nabla_{k}$ derivative of the first formula in (23) and applying (13) we obtain:

$$
\left(\lambda^{s} \delta_{k}^{i}+\lambda^{i} \delta_{k}^{s}\right) \nabla_{s} \nabla_{j} h+L^{s i} \nabla_{k} \nabla_{s} \nabla_{j} h=\nabla_{k} \rho \delta_{j}^{i}
$$


Now subtract the same relation with indices $j$ and $k$ interchanged, use the fact that $\nabla_{k} \nabla_{s} \nabla_{j} h=$ $\nabla_{j} \nabla_{s} \nabla_{k} h$ (since $g$ is flat) and substitute $\nabla_{i} \nabla_{j} h$ from the second formula in (23):

$$
\rho \lambda_{\beta}\left(L^{-1}\right)_{j}^{\beta} \delta_{k}^{i}-\rho \lambda_{\beta}\left(L^{-1}\right)_{k}^{\beta} \delta_{j}^{i}=\nabla_{k} \rho \delta_{j}^{i}-\nabla_{j} \rho \delta_{k}^{i} .
$$

This implies $\nabla_{k} \rho=-\rho \lambda_{\beta}\left(L^{-1}\right)_{k}^{\beta}$ or, equivalently, in more invariant way:

$$
\mathrm{d} \rho=-\rho\left(L^{-1}\right)^{*} \mathrm{~d} \lambda .
$$

If $\rho \equiv 0$, then (23) becomes $\nabla_{i} \nabla_{j} h=0$, i.e. $h$ itself is a Casimir of $g$. Otherwise, we use the following general property of Nijenhuis operators [3, proposition 2.2]

$$
L^{*} \mathrm{~d}(\operatorname{det} L)=2 \operatorname{det} L \mathrm{~d} \lambda,
$$

which gives $\frac{\mathrm{d} \rho}{\rho}=-\left(L^{-1}\right)^{*} \mathrm{~d} \lambda=-\frac{1}{2} \frac{\mathrm{d}(\operatorname{det} L)}{\operatorname{det} L}$ and, finally,

$$
\rho=c_{h} \frac{1}{\sqrt{\operatorname{det} L}}
$$

where $c_{h}$ is a constant function depending only on the choice of the Casimir function $h$ of $\bar{g}$. In other words, we obtain a natural map $h \mapsto c_{h}$ from the vector space of the Casimirs of $\bar{g}$ to $\mathbb{R}$. This map is obviously linear so that the common Casimirs $h$ of $\bar{g}$ and $g$ are defined by one single linear relation $c_{h}=0$, which is equivalent to the statement of the lemma.

Our next goal is to verify the second statement of theorem 2.

Lemma 4.2. Assume that $(g, L)$ is a geodesically compatible pair, the metric $g$ is flat and $\operatorname{det} L>0$. Then $\sqrt{\operatorname{det} L}$ is a Casimir of the Poisson structure corresponding to $g L^{-1}$.

Proof. We first rewrite relation (22) for Casimirs of $\bar{g}=g L^{-1}$ in a slightly different way. Taking into account that $\bar{g}^{s i}=L^{s i}$ and using (13), we obtain the following expression for the lhs of (22):

$$
\begin{aligned}
\bar{g}^{s i} \nabla_{s} \nabla_{j} h+\left(\lambda^{s} \nabla_{s} h+K h\right) \delta_{j}^{i} & =L^{s i} \nabla_{j} \nabla_{s} h+\left(\lambda^{s} \nabla_{s} h+K h\right) \delta_{j}^{i} \\
& =\nabla_{j}\left(L^{s i} \nabla_{s} h\right)-\left(\nabla_{j} L^{\mathrm{si}}\right) \nabla_{s} h+\left(\lambda^{s} \nabla_{s} h+K h\right) \delta_{j}^{i} \\
& =\nabla_{j}\left(L^{s i} \nabla_{s} h\right)-\left(\lambda^{s} \delta_{j}^{i}+\lambda^{i} \delta_{j}^{s}\right) \nabla_{s} h+\left(\lambda^{s} \nabla_{s} h+K h\right) \delta_{j}^{i} \\
& =\nabla_{j}\left(L^{s i} \nabla_{s} h\right)-\lambda^{i} \nabla_{j} h+K h \delta_{j}^{i}
\end{aligned}
$$

Thus, we need to verify that $h=\sqrt{\operatorname{det} L}$ satisfies

$$
\nabla_{j}\left(L^{s i} \nabla_{s} h\right)-\lambda^{i} \nabla_{j} h+K h \delta_{j}^{i}=0 .
$$

Once again we use the general property (24) of Nijenhuis operators that gives $L_{r}^{s} \nabla_{s} \sqrt{\operatorname{det} L}=$ $\lambda_{r} \sqrt{\operatorname{det} L}$ or, equivalently, $L^{s i} \nabla_{s} \sqrt{\operatorname{det} L}=\lambda^{i} \sqrt{\operatorname{det} L}$. Hence, for $h=\sqrt{\operatorname{det} L}$ we have:

$$
\begin{aligned}
& \nabla_{j}\left(L^{s i} \nabla_{s} \sqrt{\operatorname{det} L}\right)-\lambda^{i} \nabla_{j} \sqrt{\operatorname{det} L}+K \sqrt{\operatorname{det} L} \delta_{j}^{i} \\
& =\nabla_{j}\left(\lambda^{i} \sqrt{\operatorname{det} L}\right)-\lambda^{i} \nabla_{j} \sqrt{\operatorname{det} L}+K \sqrt{\operatorname{det} L} \delta_{j}^{i} \\
& =\lambda^{i} \nabla_{j} \sqrt{\operatorname{det} L}+\left(\nabla_{j} \lambda^{i}\right) \sqrt{\operatorname{det} L}-\lambda^{i} \nabla_{j} \sqrt{\operatorname{det} L}+K \sqrt{\operatorname{det} L} \delta_{j}^{i} \\
& =\left(\nabla_{j} \lambda^{i}+K \delta_{j}^{i}\right) \sqrt{\operatorname{det} L}=0, \\
& 5148
\end{aligned}
$$


as required (at the very last step we used lemma 3.4).

The third statement of theorem 2 now immediately follows from lemmas 4.1 and 4.2. Indeed for any Casimir of $\bar{g}$, and in particular for $h=\sqrt{\operatorname{det} L}$, formulas (23) and (25) give:

$$
\nabla^{i} \nabla_{j} h=\frac{c_{h}}{\sqrt{\operatorname{det}(L)}}\left(L^{-1}\right)_{j}^{i}
$$

It remains to replace $L$ with the linear combination $-\alpha L+$ Id.

\section{Proof of theorem 3 and corollary 2.1 and more properties of the related pencils}

Proof of Theorem 3. Let $x^{1}, \ldots, x^{n}$ be flat coordinates for the metric $g$. In these coordinates, the Christoffel symbols vanish so that the covariant derivative coincides with the usual one. Then formula (17) reads

$$
\frac{\partial \lambda^{i}}{\partial x^{j}}=-K \delta_{j}^{i}
$$

with a constant $K$. Hence,

$$
\lambda^{i}=b^{i}-K x^{i}
$$

for some constants $b^{1}, \ldots, b^{n}$.

Next, using the fact that $\nabla_{k}=\frac{\partial}{\partial x^{k}}$, we obtain from (13):

$$
\frac{\partial}{\partial x^{k}} L^{i j}=\lambda^{i} \delta_{k}^{j}+\lambda^{j} \delta_{k}^{i(29)}=\left(b^{i}-K x^{i}\right) \delta_{k}^{j}+\left(b^{j}-K x^{j}\right) \delta_{k}^{j}
$$

This system of equations implies $L^{i j}=a^{i j}+b^{i} x^{j}+b^{j} x^{i}-K x^{i} x^{j}$ (with constants $a^{i j}$ that are necessarily symmetric with respect to $i$ and $j$ ) as we claimed. Clearly every such $L^{i j}$ satisfies (30) so it is geodesically compatible with $g$.

To finish the proof, recall that near the points where $L^{i j}$ is non-degenerate, the metric $g L^{-1}$ has constant curvature $K$ by lemma 3.4, and that $L^{i j}=g^{i s} L_{s}^{j}$ is the contravariant inverse of $\bar{g}=g L^{-1}$. Theorem 3 is proved.

Proof of Corollary 2.1. As above, $g$ denotes a flat metric and $L$ is an operator geodesically compatible with $g$. Let $P$ and $Q$ be polynomials such that $P(L)$ and $Q(L)$ are non-degenerate. The operator connecting the metrics $g P(L)^{-1}$ and $g Q(L)^{-1}$ is a rational function of the Nijenhuis operator $L$ and, therefore, is Nijenhuis also by [3, proposition 3.1], so the first condition from the definition of compatibility is fulfilled. We need to prove the 'curvature-additivity' condition (8).

In order to do it, we observe that in local coordinates from theorem 3 , the curvature tensors of $g P(L)^{-1}$ and $g Q(L)^{-1}$ are given by rational functions in

$$
N:=n+\frac{(n+1) n}{2}+\frac{(n+1)(n+2)}{2}
$$

variables: the first $n$ variables are $x^{1}, \ldots, x^{n}$, the next $\frac{(n+1) n}{2}$ are $g^{i j}$ and the last $\frac{(n+1)(n+2)}{2}$ are the data $\left(a^{i j}, b^{i}, K\right)$. The 'curvature-additivity' condition (8) is then equivalent to a system of algebraic relations on these $N$ variables. If it is fulfilled in an non-empty open subset of $\mathbb{R}^{N}$, it is fulfilled everywhere. Let us explain why such a subset exists. 
Recall that by $[29,30]$ if two metrics $g$ and $\bar{g}$ are related by a Nijenhuis operator $L=L_{j}^{i}=$ $\bar{g}^{\text {is }} g_{s j}$ with $n=\operatorname{dim} M$ distinct real eigenvalues, then the curvature tensors of $g$ and $\bar{g}$ satisfy (8) automatically ${ }^{4}$. This statement also follows from our proof of theorem 5 , see remark 7.2 below. Next, notice that (9) gives us an example of $L$ having $n$ different real eigenvalues. More precisely, let $\check{g}_{i j}, \check{a}^{i j}, \breve{b}^{i}$ and $\breve{K}$ be the data from theorem 3 corresponding to the example (9) and at some point $\check{x} \in \mathbb{R}^{n}$ the corresponding $L$ has $n$ different real eigenvalues. At every point of a small neighborhood of $\left(\check{x}^{i}, \check{g}_{i j}, \check{a}^{i j}, \check{b}^{i}, \check{K}\right)$ in $\mathbb{R}^{N}$, the corresponding operator $L$ still satisfies the property that its eigenvalues are real and different. Using the above mentioned result from $[29,30]$, we conclude that in this neighbourhood, (8) is fulfilled which implies that it is fulfilled identically for all $L$ and at all points whenever it makes sense.

We conclude this section with discussing some more properties of the pencil $\mathcal{L}_{g}$ of Nijenhuis operators $L_{j}^{i}$ geodesically equivalent to $g$ (equivalently, pencil $\mathcal{L}$ of Poisson compatible contravariant metrics $L^{i j}$ ) from theorem 3. Notice that the relation between these two pencils can be written as $\mathcal{L}_{g}=\mathcal{L} g^{-1}$. Also notice that $\mathcal{L}$ does not depend on the choice of $g$ whereas $L_{g}$ does.

First observe that $\operatorname{dim} \mathcal{L}_{g}$ equals $\frac{n(n+1)}{2}+n+1=\frac{(n+2)(n+1)}{2}$, which is the dimension of the space of symmetric $(n+1) \times(n+1)$-matrices. As the next theorem shows, this is not a coincidence: to each $L^{i j}$ of form (7) one can uniquely assign such a matrix by a natural geometric procedure.

Proposition 5.1. Consider $\mathbb{R}_{>0} \times \mathbb{R}^{n}$ (the coordinate on $\mathbb{R}_{>0}$ will be denoted by $x^{0}$ and those on $\mathbb{R}^{n}$ will be $\left.x^{1}, \ldots, x^{n}\right)$ with the symmetric affine connection $\hat{\nabla}=\left(\hat{\Gamma}_{\beta \gamma}^{\alpha}\right)$ (with $\alpha, \beta, \gamma \in$ $\{0, \ldots, n\})$ such that the only non-zero Christoffel symbols are as follows:

$$
\hat{\Gamma}_{j 0}^{i}=\hat{\Gamma}_{0 j}^{i}=\delta_{j}^{i} \frac{1}{x^{0}} \quad \text { for } i, j=1, \ldots, n
$$

Then $\hat{\nabla}$ is flat. Moreover, for any constants $\left(a^{i j}, b^{i}, K\right)$ with $i, j=1, \ldots, n$ and $a^{i j}=a^{j i}$, the symmetric contravariant $(2,0)$ tensor $A^{\alpha \beta}$ on $\mathbb{R}_{>0} \times \mathbb{R}^{n}$ given by

$$
A^{i j}=\frac{1}{\left(x^{0}\right)^{2}} L^{i j}, \quad A^{0 i}=-\frac{b^{i}-K x^{i}}{x^{0}}, \quad A_{00}=-K \quad(i, j=1, \ldots, n),
$$

is parallel with respect to $\hat{\nabla}$. Furthermore, in the 'new' coordinates

$$
y^{0}=x^{0}, \quad y^{i}=x^{0} x^{i} \quad(i=1, \ldots, n),
$$

the Christoffel symbols of this connection vanish. In these coordinates, the matrix of A becomes constant with components

$$
A^{i j}=a^{i j}, \quad A^{0 i}=A^{i 0}=-b^{i}, \quad A_{00}=-K \quad(i, j=1, \ldots, n) .
$$

Proof. One verifies proposition 5.1 by direct calculations. First we substitute the Christoffel symbols (32) into the formula (45) for the curvature tensor and see that it vanishes. Next we substitute (33) in the relation $\hat{\nabla} A=0$ and see that it is equivalent to (30). Again by direct

\footnotetext{
${ }^{4}$ Conversely, the 'curvature-additivity' condition (8) becomes essential in the case of operators with multiple eigenvalues, which do appear in our setting, so that the direct statement from [29, 30] is not formally applicable here.
} 
calculations we observe that $\hat{\nabla}$-Hessians of the functions $y^{\alpha}$ vanish and, therefore, in the $y^{\alpha}$ coordinates the components of $A$ are constants. Finally, calculating the Jacobi matrix $\left(\frac{\partial y}{\partial x}\right)$ and using it in the transformation rule for $(2,0)$-tensors finishes the proof.

Note that the construction of the connection $\hat{\nabla}$ on $\mathbb{R}_{>0} \times \mathbb{R}^{n}$ does not involve $g$ or $L$, so it is the same for all $L$ and all $g$ from theorem 3. In fact it is motivated by different 'conification' procedures in projective (see e.g. $[14,16,18]$ ) and other Cartan parabolic geometries.

Proposition 5.1 reduces the classification of geodesically compatible pairs $(g, L)$ with flat $g$ to the classification of pairs of symmetric $(n+1) \times(n+1)$-matrices which is, of course, well known (see e.g. [25]). Note that the metric $g$ itself is included in the family (7) from theorem 3 with $L=\mathrm{Id}$, i.e., $a^{i j}=g^{i j}, b^{i}=0$ and $K=0$. We see that the first column and first row of the corresponding matrix $A_{g}$ vanish, so that in terms of [25] we are only interested in those pairs $A_{g}, A \in \operatorname{Sym}((n+1) \times(n+1))$ for which $A_{g}$ has rank $n$.

Our next observation is maximality of the pencils $\mathcal{L}_{g}$ and $\mathcal{L}$ and their relations with other (simpler) pencils. Let $x^{1}, \ldots, x^{n}$ be canonical coordinates for the Euclidean metric $g \simeq \sum \mathrm{d}\left(x^{i}\right)^{2}$. Consider the family $\mathcal{S}$ of operators $A$ given in these coordinates by symmetric matrices with constant entries. Obviously every $A \in \mathcal{S}$ is a Nijenhuis operator and therefore $\mathcal{S}$ is a Nijenhuis pencil.

Can one extend $\mathcal{S}$ to get a larger Nijenhuis pencil? To answer this question, we need to describe Nijenhuis operators $L$ such that for each $A \in \mathcal{S}$ the sum $L+A$ is still Nijenhuis. Analytically, this condition means that $L$ commutes with all $A \in \mathcal{S}$ in sense of Frolicher-Nijenhuis bracket [15], that is,

$$
\begin{aligned}
{[[L, A]](\xi, \eta)=} & L A[\xi, \eta]-L[A \xi, \eta]-L[\xi, A \eta]+[L \xi, A \eta] \\
& +A L[\xi, \eta]-A[L \xi, \eta]-A[\xi, L \eta]+[A \xi, L \eta]=0
\end{aligned}
$$

for any vector fields $\xi$ and $\eta$. If we temporarily ignore the fact that $L$ itself is a Nijenhuis operator, then the problem becomes linear and can be solved by straightforward computation that we omit.

Proposition 5.2. Let $[[L, A]]=0$ for all $A \in \mathcal{S}$. Then for $n \geqslant 3$, in the coordinates $x^{1}, \ldots, x^{n}$, the matrix of $L$ takes the following form:

$$
L=A+x b^{\top}+c x^{\top}+K x x^{\top}, \quad \text { where } x=\left(\begin{array}{c}
x^{1} \\
\vdots \\
x^{n}
\end{array}\right), \quad b=\left(\begin{array}{c}
b^{1} \\
\vdots \\
b^{n}
\end{array}\right), \quad c=\left(\begin{array}{c}
c^{1} \\
\vdots \\
c^{n}
\end{array}\right),
$$

$A$ is an arbitrary constant matrix and $K \in \mathbb{R}$ (cfformula (7) from theorem 3$)$.

In dimension 2, $L$ is the sum of an operator (35) and arbitrary skew-symmetric operator of the form $\left(\begin{array}{cc}0 & f\left(x^{1}, x^{2}\right) \\ -f\left(x^{1}, x^{2}\right) & 0\end{array}\right)$.

Notice that in order for (35) to be a Nijenhuis operator, the parameters $A, b, c$ and $K$ must satisfy additional (nonlinear) relations. However, the following fact is straightforward.

Corollary 5.1. There is a unique Nijenhuis pencil $\mathcal{L}_{g}$ satisfying the following conditions:

(a) $\mathcal{L}_{g}$ is maximal,

(b) $\mathcal{L}_{g}$ contains $\mathcal{S}$,

(c) all operators from $\mathcal{L}_{g}$ are g-symmetric. 
The matrices of operators $L \in \mathcal{L}_{g}$ in coordinates $x^{1}, \ldots, x^{n}$ are given by (35) with $b=c$ and symmetric $A$. In other words, $\mathcal{L}_{g}$ is the Nijenhuis pencil from theorem 3 with $g_{i j}=\delta_{i j}$.

If we omit condition (c), then there exist other maximal Nijenhuis extensions of $\mathcal{S}$. For instance, we may take all operators $L$ of the form $L(x)=A+x b^{\top}$ with arbitrary constant $A$ (not necessarily symmetric!).

For a flat metric $g$ of arbitrary signature, corollary 5.1 can be generalized as follows. Let $\mathcal{S}$ be a Nijenhuis tensor that consists of covariantly constant $g$-symmetric operators $A$, then there exists a unique Nijenhuis pencil $\mathcal{L}_{g}$ satisfying the above conditions (a)-(c). This pencil coincides with that from theorem 3 . The maximality of $\mathcal{L}_{g}$ immediately implies

Corollary 5.2. The pencil $\mathcal{L}$ of Poisson-compatible metrics from theorem 3 is maximal.

This pencil admits the following alternative description. Let $g$ be a flat metric and $\nabla$ be its Levi-Civita connection. Consider all the metrics $\hat{g}$ covariantly constant w.r.t. $\nabla$. Obviously, they are flat and pairwise Poisson-compatible.

Corollary 5.3. Assume that $\bar{g}$ is almost compatible with every covariantly constant $\hat{g}$ in the sense of $[29,30]$, i.e., $L=\bar{g}^{-1} \hat{g}$ is Nijenhuis. Then $\bar{g}$ has constant curvature. Moreover, any two metrics $\bar{g}_{1}$ and $\bar{g}_{2}$ satisfying this condition are Poisson compatible. All together they form the pencil $\mathcal{L}$ from theorem 3.

\section{Proof of theorem 4 and Poisson compatibility of metrics $g, g L^{-1}, g L^{-2}, \ldots$}

Throughout this section, $g$ denotes a flat metric and $L$ is an operator geodesically compatible with $g$.

From lemma 3.4 (and theorem 3) we see that the metric $g L^{-1}$ is flat if and only if $K=0$. The next two statements answer the natural question on necessary and sufficient conditions for the metric $g L^{-2}$, and more generally, all the metrics $g L^{-k}$ with $k \leqslant k_{0}$ to be flat.

Lemma 6.1. Suppose $g$ is flat and $L$ is geodesically compatible with $g$ and non-degenerate. Let $g L^{-1}$ be flat, then $\lambda^{j}$ is a parallel vector field, i.e., $\nabla_{i} \lambda^{j}=0$, implying that $g^{i j} \lambda_{i} \lambda_{j}$ is a constant. Moreover, the metric $g L^{-2}$ is flat if and only if $\lambda^{j}$ is null, that is, $g_{i j} \lambda^{i} \lambda^{j}=g^{i j} \lambda_{i} \lambda_{j}=0$.

Proof. Since $g L^{-1}$ is flat, $K$ in lemma 3.4 is zero. Hence, (17) implies that $\lambda^{i}$ is $\nabla$-parallel. Of course, the same is seen from theorem 3 , since in this case $\lambda^{i}=a^{i}$ in flat coordinates and is clearly parallel.

In order to prove the second claim, we use (17) for the metric $\bar{g}=g L^{-1}$ which is still compatible with $L: \bar{\nabla}^{i} \lambda_{j}+\bar{K} \delta_{j}^{i}=0$. In view of (15), we have $\bar{\nabla}^{i} \lambda_{j}=L_{s}^{i} \nabla^{s} \lambda_{j}-\lambda^{s} \lambda_{s} \delta_{j}^{i}$. Since $\nabla^{s} \lambda_{j}=\nabla_{j} \lambda^{s}=0$, we see that $\bar{K}=0$ if and only if $\lambda^{s} \lambda_{s}=g^{i s} \lambda_{i} \lambda_{s}=0$.

Corollary 6.1. Suppose $g$ is flat, L is geodesically compatible with $g$ and non-degenerate.

The necessary and sufficient conditions for flatness of each of the metrics $g, g L^{-1}, \ldots, g L^{-k}$ $(k \geqslant 2)$ are as follows: the one-form $\mathrm{d}\left(\frac{1}{2} \operatorname{tr} L\right)=\lambda_{i}$ is parallel with respect to $\nabla=\nabla^{g}$ and

$$
g^{i j} \lambda_{i} \lambda_{j}=\left(g L^{-1}\right)^{i j} \lambda_{i} \lambda_{j}=\cdots=\left(g L^{-k+2}\right)^{i j} \lambda_{i} \lambda_{j}=0 .
$$

Moreover, if each of the metrics $g, g L^{-1}, \ldots, g L^{-k}(k \geqslant 2)$ is flat, then $\lambda_{i}$ is parallel with respect each Levi-Civita connection related to $g, g L^{-1}, \ldots, g L^{-k+1}$.

Here by $\left(g L^{-m}\right)^{i j}$ we denote the dual tensor to $\left(g L^{-m}\right)_{i j}$. In other words, in matrix notation, $\left(g L^{-m}\right)^{i j}$ are the components of the inverse matrix $\left(g L^{-m}\right)^{-1}=L^{m} g^{-1}$.

Proof. Corollary 6.1 follows from iterative application of lemma 6.1. 
This corollary allows us to construct examples of pairs $(g, L)$ such that $g L^{-k}$ is flat for all $k>0$ and $\operatorname{d} \operatorname{tr}(L) \neq 0$. The simplest example of this kind is as follows. Let $b=b^{i} \neq 0$ be a constant vector which is null with respect to a non-degenerate symmetric matrix $g_{i j}$. We view $g$ as a flat metric and take $a^{i j}=\left(g^{-1}\right)$ and $K=0$. The $(2,0)$-tensor $L^{i j}$ constructed by (7) for these $a^{i j}, b^{i}, K$ evidently has the property that $\lambda^{i}$ is parallel with respect to $\nabla^{g}$ and that $\left(g L^{-k}\right)^{i j} \lambda_{i} \lambda_{j}=0$ for every $k$.

Let us give a more interesting example based on the same linear-algebraic idea.

Example 6.1. Take

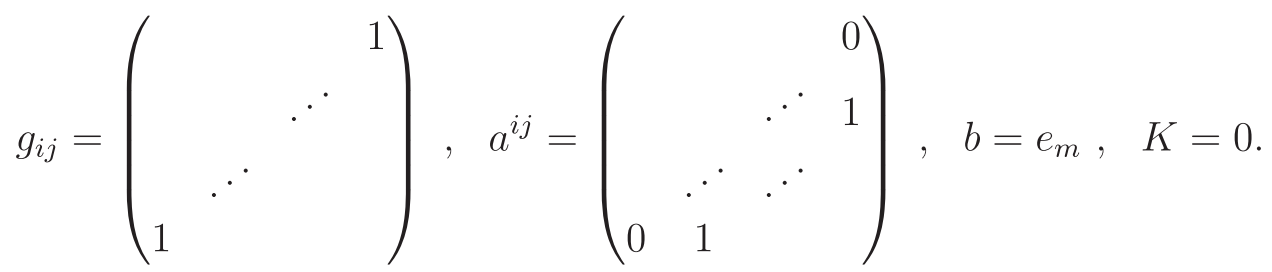

(Here $e_{m}$ denotes the $m$ th basis vector so that all components of $b$ vanish except for the $m$ th one which is equal to 1 ). Now if $m \geqslant n / 2+1$, then the necessary and sufficient flatness conditions for $g, g L^{-1}, \ldots, g L^{-k}$ from corollary 6.1 are fulfilled for any $k$. This implies, in particular, that for any real analytic function $f$ of one variable, the metric $g(f(L))^{-1}$ is also flat (at those points where $f(L)$ is non-degenerate) so that we obtain an infinite-dimensional pencil of contravariant flat metrics of the form $f(L) g^{-1}$.

This example may possibly be of interest in the study of infinite-dimensional systems of hydrodynamic type for the following reason. In this theory one customary assumes that the operators defining these systems are diagonalizable and this special case is well studied. The case of other Segre characteristics is generally considered to be much harder and, in fact, there are only very few such examples in the literature, see e.g. [2, 10, 33, 37]. In example 6.1, the corresponding operator $L_{j}^{i}=L^{i s} g_{s j}$ has nontrivial Jordan blocks. For instance, in the fourdimensional case with $m=3$ and $g$ and $L$ given by:

$$
g_{i j}=\left(\begin{array}{cccc} 
& & & 1 \\
& & 1 & \\
& 1 & & \\
1 & & &
\end{array}\right), \quad L^{i j}=\left(\begin{array}{cccc}
0 & 0 & x^{1} & 0 \\
0 & 0 & x^{2} & 1 \\
x^{1} & x^{2} & 2 x^{3}+1 & x^{4} \\
0 & 1 & x^{4} & 0
\end{array}\right),
$$

the operator $L_{j}^{i}$ has two Jordan $2 \times 2$ blocks with nonconstant eigenvalues. After a suitable coordinate transformation, $g$ and $L$ become

$$
\begin{aligned}
g_{i j} & =\left(\begin{array}{cccc}
2\left(-2 x^{2}-1\right)\left(x^{3}-x^{1}\right) & \left(x^{3}-x^{1}\right)^{2} & 0 & 0 \\
\left(x^{3}-x^{1}\right)^{2} & 0 & 0 & 0 \\
0 & 0 & 2\left(-2 x^{4}-1\right)\left(x^{1}-x^{3}\right) & \left(x^{1}-x^{3}\right)^{2} \\
0 & & 0 & \left(x^{1}-x^{3}\right)^{2}
\end{array}\right) \\
L_{j}^{i} & =\left(\begin{array}{cccc}
x^{1} & 0 & 0 & 0 \\
2 x^{2}+1 & x^{1} & 0 & 0 \\
0 & 0 & x^{3} & 0 \\
0 & 0 & 2 x^{4}+1 & x^{3}
\end{array}\right) .
\end{aligned}
$$


This phenomenon survives for all dimensions. The appearing Jordan blocks are $2 \times 2$, the number of such blocks depends on $m$ and in the case of even $n$ and $m=n / 2+1$ equals $n / 2$. The corresponding eigenvalues are not constant.

As far as we know, such examples (with many Jordan blocks with nonconstant eigenvalues) were not known before and may open a door to other Segre characteristics in the theory of integrable systems of hydrodynamic type.

Now, if in example 6.1 we set $m<n / 2+1$, then it is a simple exercise in linear algebra to check that for a certain $k_{0}$, the metric $g L^{-k_{0}-1}$ has constant non-zero curvature, whereas $g, g L^{-1}, \ldots, g L^{-k_{0}}$ are still flat. The maximal value of such $k_{0}$ is $n$. Let us give the corresponding example.

Example 6.2. On $\mathbb{R}^{n}$ with coordinates $x^{1}, \ldots, x^{n}$, consider the metric $g$ and the tensor $L^{i j}$ given by (7) with the following data $A, b$ and $K$ :

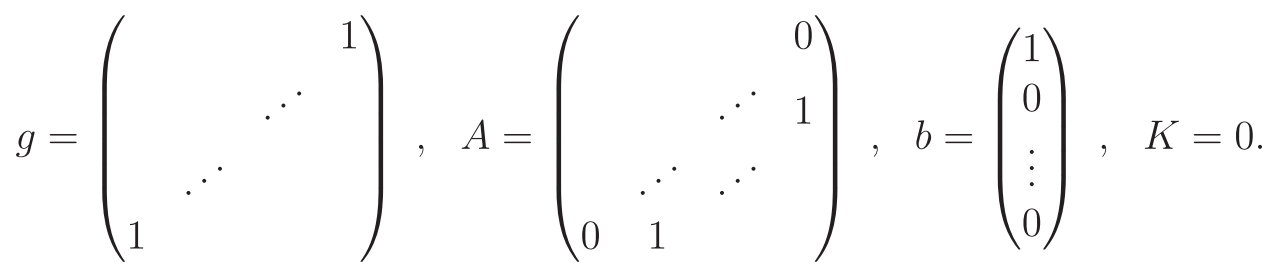

Then the metrics $g L^{-1}, \ldots, g L^{-n}$ are flat and $g L^{-n-1}$ has constant non-zero curvature.

In this example $L_{j}^{i}$ has $n$ different nonconstant eigenvalues at a generic point. From theorem 5 it follows that this pair $(g, L)$ is locally isomorphic (modulo multiplication of $L$ by a constant) to that from (10) (or (9), at almost every point).

Proof of Theorem 4. We assume that $g$ is Einstein with a constant non-zero scalar curvature, $L$ is geodesically compatible to $g$, $\operatorname{det} L>0$ and $\operatorname{d} \operatorname{tr}(L) \neq 0$. By $\tilde{g}$ we denote the metric $(\operatorname{det} L)^{-1} g L^{-1}$ that is geodesically equivalent to $g$.

Note that $\tilde{g}$ is also Einstein (with constant scalar curvature). In dimension $n>2$ it follows from [23, lemma 3 and corollary 5], and in dimension $n=2$ it is Beltrami theorem (see e.g. [27]).

Next, consider the function $\phi=-\log \sqrt{\operatorname{det} L}$ and $\phi_{i}=\nabla_{i} \phi=-\mathrm{d}(\log \sqrt{\operatorname{det} L})$. It is known (e.g., [23, section 2.2]) that the Christoffel symbols and Ricci tensors of $\tilde{g}$ and $g$ are related by the following formulas:

$$
\begin{aligned}
& \tilde{\Gamma}_{j k}^{i}=\Gamma_{j k}^{i}+\delta_{j}^{i} \phi_{k}+\delta_{k}^{i} \phi_{j} . \\
& \tilde{R}_{i j}=R_{i j}-(n-1)\left(\nabla_{j} \phi_{i}-\phi_{i} \phi_{j}\right) .
\end{aligned}
$$

Replacing the $g$-covariant derivative $\nabla$ in (37) by $\tilde{g}$-covariant derivative $\tilde{\nabla}$, we obtain

$$
\tilde{R}_{i j}=R_{i j}-(n-1)\left(\tilde{\nabla}_{j} \phi_{i}+\phi_{i} \phi_{j}\right) .
$$

Next, making the substitution $\phi=\log \psi\left(\right.$ in our case $\psi=(\sqrt{\operatorname{det} L})^{-1}$ ), we see that

$$
\tilde{\nabla}_{j} \phi_{i}+\phi_{i} \phi_{j}=\tilde{\nabla}_{j} \frac{\tilde{\nabla}_{i} \psi}{\psi}+\frac{\tilde{\nabla}_{i} \psi}{\psi} \frac{\tilde{\nabla}_{j} \psi}{\psi}=\frac{1}{\psi} \tilde{\nabla}_{j} \tilde{\nabla}_{i} \psi
$$

Note that the metric $\bar{g}=g L^{-1}$ we are interesting in is conformally related to $\tilde{g}=$ $(\text { det } L)^{-1} g L^{-1}$ and the conformal coefficient is $\psi^{-2}=\mathrm{e}^{-2 \phi}$. It is well-known (see for example 
[9, equation (2.21)]) that the Ricci tensors $\tilde{R}_{i j}$ and $\bar{R}_{i j}$ of two conformally related metrics $\tilde{g}$ and $\bar{g}=\psi^{-2} \tilde{g}=\mathrm{e}^{-2 \phi} \tilde{g}$ are connected by

$$
\bar{R}_{i j}=\tilde{R}_{i j}+\left(\Delta_{\tilde{g}} \phi-(n-2)\|\tilde{\nabla} \phi\|_{\tilde{g}}^{2} \tilde{g}_{i j}+\frac{n-2}{\psi} \tilde{\nabla}_{i} \tilde{\nabla}_{j} \psi .\right.
$$

Here $\Delta_{\tilde{g}} \phi:=\tilde{g}^{i j} \tilde{\nabla}_{i} \tilde{\nabla}_{j} \phi$ is just the $\tilde{g}$-Beltrami-Laplace operator applied to $\phi$.

Starting from this point, our proof depends on dimension. We first consider the case $n>2$. In this case the first two terms on the right-hand side of (40) are proportional to $\tilde{g}$. If $\bar{g}$ is Einstein, the left-hand side is also proportional to $\tilde{g}$, so $\tilde{\nabla}_{i} \tilde{\nabla}_{j} \psi$ is proportional to $\tilde{g}$. But this leads to a contradiction with (38) and (39). Indeed, then $R_{i j}$ is proportional to $\tilde{g}_{i j}$. Hence, $g_{i j}$ is proportional to $\tilde{g}_{i j}$, so $L_{j}^{i}$ is proportional to $\delta_{j}^{i}$. By the Weyl theorem [38], this implies that $\mathrm{d} \operatorname{tr} L=0$ which is forbidden by our assumptions.

Let us now consider the remaining dimension $n=2$. From corollaries 1 and 2 of [23] we see that if a metric $g$ is Einstein of constant scalar curvature $(n-1) K$ and $L$ is compatible with it, then in addition to (12) the following equation holds for a certain constant $C$ :

$$
\nabla^{i} \lambda_{j}=(-K \operatorname{tr} L+C) \delta_{j}^{i}-K L_{j}^{i} .
$$

Note that though in [23] one generally assumes that $n=\operatorname{dim} M \geqslant 3$, formula (41) holds in dimension 2 as well; in fact the statement $\nabla^{i} \lambda_{j}=\mu \delta_{j}^{i}-K L_{j}^{i}$ (which is corollary 1 in [23]) follows from and is equivalent to (37), and the proof of the relation $\nabla_{i} \mu=-2 K \lambda_{i}$, which is corollary 2 in [23], is straightforward and works also in dimension 2. Note also that (41) for metrics of constant curvature follows directly from [14, theorem 5.1] or from [35, theorem 1].

Next, in view of lemma 3.1 we have

$$
\bar{g}^{s i} \bar{\nabla}_{s} \lambda_{j}=L_{s}^{i} \nabla^{s} \lambda_{j}+\lambda^{s} \lambda_{s} \delta_{j}^{i(41)}=(C-K \operatorname{tr} L) L_{j}^{i}-K\left(L^{2}\right)_{j}^{i} .
$$

Now we use the fact that in dimension 2, we have $\left(L^{2}\right)_{j}^{i}-\operatorname{tr} L \cdot L_{j}^{i}+\operatorname{det} L \cdot \delta_{j}^{i}=0$ for any operator $L$ (Cayley-Hamilton theorem). This allows us to replace $\left(L^{2}\right)_{j}^{i}$ in the above formula by $\operatorname{tr} L \cdot L_{j}^{i}-\operatorname{det} L \cdot \delta_{j}^{i}$ and we obtain

$$
\bar{g}^{s i} \bar{\nabla}_{s} \lambda_{j}=\left(\lambda^{s} \lambda_{s}+K \operatorname{det} L\right) \delta_{j}^{i}+(C-2 K \operatorname{tr} L) L_{j}^{i} .
$$

We see that the left-hand side of this formula is as in the formula (41) written for the metric $\bar{g}$; this implies that $(-2 K \operatorname{tr} L+C)=$ const. which is forbidden by the assumptions $K \neq 0$ and $\mathrm{d} \operatorname{tr} L \neq 0$. The obtained contradiction proves theorem 4 .

Combining theorem 4 with lemma 6.1 we immediately see that for positive definite flat metrics the number $k_{0}$ such that $g, g L^{-1}, \ldots, g L^{-k_{0}}$ are flat is at most two and the number $k_{0}$ such that $g, g L^{-1}, \ldots, g L^{-k_{0}}$ are of constant curvature is at most three.

Corollary 6.2. Suppose $g$ is a Euclidean metric (i.e., flat and positive definite). Let $L$ be geodesically compatible with $g$, non-degenerate and $\mathrm{d} \operatorname{tr} L \neq 0$.

Then there is the following alternative: either $g L^{-1}$ has constant non-zero curvature and $g L^{-2}$ is not of constant curvature, or $g L^{-1}$ is flat, $g L^{-2}$ has constant non-zero curvature and $g L^{-3}$ is not of constant curvature. In particular, $g L^{-2}$ cannot be flat.

Proof. If $\lambda_{i}$ is parallel, then in the positively definite case it is not null (recall that the condition $\mathrm{d} \operatorname{tr} L \neq 0$ implies that $\lambda_{i} \neq 0$ ). Applying lemma 6.1 we obtain the required claim. 


\section{Proof of theorem 5}

Proof. Suppose $L$ is differentially-non-degenerate, then at almost every point it has $n$ different eigenvalues. We will work in a small neighbourhood of such a point and first consider the case when the eigenvalues are real. In this case, there exists a coordinate system $x^{1}, \ldots, x^{n}$ such that $L$ is given by

$$
\operatorname{diag}\left(x^{1}, x^{2}, \ldots, x^{n}\right) .
$$

Let the metrics $g L^{-k}$ with $k \in\{0, \ldots, n+1\}$ satisfy the assumptions of theorem 5 . Since the $(0,2)$-tensor $g L^{-1}$ is symmetric, $g$ is diagonal:

$$
g:=\operatorname{diag}\left(g_{1}, g_{2}, \ldots, g_{n}\right),
$$

where $g_{i}=g_{i}\left(x^{1}, \ldots, x^{n}\right)$ are some functions. Let us show that in this coordinate system the metric is given (up to a constant) by (9), which implies that $L$ is geodesically compatible to $g$.

We need the following technical lemma:

Lemma 7.1. Let $g$ be diagonal as in (43) and

$$
L=\operatorname{diag}\left(h_{1}\left(x^{1}\right), h_{2}\left(x^{2}\right), \ldots, h_{n}\left(x^{n}\right)\right)
$$

with the function $h_{i}$ depending on the coordinate $x^{i}$ only.

Then the Christoffel symbols $\Gamma_{j k}^{i}$ of the metric $g$ are as follows:

- $\Gamma_{i j}^{k}=0$ for pairwise different $i, j$ and $k$,

- $\Gamma_{k j}^{k}=\frac{1}{2} \frac{1}{g_{k}} \frac{\partial g_{k}}{\partial x^{j}}$ for arbitrary $k, j$,

- $\Gamma_{j j}^{k}=-\frac{1}{2} \frac{1}{g_{k}} \frac{\partial g_{j}}{\partial x^{k}}$ for arbitrary $k \neq j$.

Consequently, the Christoffel symbols $\bar{\Gamma}_{j k}^{i}$ of the metric $\bar{g}=g L^{-1}$ are as follows:

- $\bar{\Gamma}_{i j}^{k}=0$ for pairwise different $i, j$ and $k$,

- $\bar{\Gamma}_{k j}^{k}=\Gamma_{k j}^{k}$ for arbitrary $k \neq j$,

- $\bar{\Gamma}_{j j}^{k}=\frac{h_{k}}{h_{j}} \Gamma_{j j}^{k}$ for arbitrary $k \neq j$,

- $\bar{\Gamma}_{k k}^{k}=\Gamma_{k k}^{k}-\frac{1}{2} \frac{1}{h_{k}} h_{k}^{\prime}$ for arbitrary $k$,

(each $h_{k}$ is a function of one variable, so $h_{k}^{\prime}$ in the latter formula and below, in e.g. (44), is just the usual derivative, $h_{k}^{\prime}=\frac{\partial h_{k}}{\partial x^{k}}$ ).

We leave the proof as a simple exercise for the reader. One needs to substitute the components of $g$ in the formula $\Gamma_{j k}^{i}=\frac{1}{2} g^{s i}\left(\frac{\partial g_{s k}}{\partial x^{j}}+\frac{\partial g_{s j}}{\partial x^{k}}-\frac{\partial g_{j k}}{\partial x^{s}}\right)$ for the Christoffel symbols of $g$ and then look how multiplications of $g_{i}$ with $1 / h_{i}$ affects the formula.

Lemma 7.2. The components of the curvature tensors $R_{j k \ell}^{i}$ and $\bar{R}_{j k \ell}^{i}$ of the connections $\Gamma$ and $\bar{\Gamma}$ from lemma 7.1 are as follows: (no summation over repeating indices)

- $R_{i j k}^{s}=0$ for arbitrary pairwise different $i, j, k$ and $s$,

- $R_{i j k}^{i}=0$ for arbitrary $i, j, k$,

- $R_{i j k}^{j}=-\frac{\partial \Gamma_{i j}^{j}}{\partial x^{k}}+\Gamma_{j i}^{j} \Gamma_{i k}^{i}+\Gamma_{j k}^{j} \Gamma_{i k}^{k}-\Gamma_{k j}^{j} \Gamma_{i j}^{j}$ for arbitrary $j \neq i$ and $i \neq k$,

- $R_{i j i}^{j}=\frac{\partial \Gamma_{i i}^{j}}{\partial x^{j}}-\frac{\partial \Gamma_{i j}^{j}}{\partial x^{i}}+\sum^{n} \Gamma_{j \alpha}^{j} \Gamma_{i i}^{\alpha}-\Gamma_{i i}^{j} \Gamma_{i j}^{i}-\Gamma_{i j}^{i} \Gamma_{i j}^{i}$ for arbitrary $i$ and $j$,

- $R_{i j i}^{k}=-R_{i i j}^{k}=\frac{\partial \Gamma_{i j}^{k}}{\partial x^{j}}+\bar{T}_{j j}^{1 k} \Gamma_{i i}^{j}+\Gamma_{j k}^{k} \Gamma_{i i}^{k}-\Gamma_{i i}^{k} \Gamma_{i j}^{i}$ for arbitrary $k \neq i$ and $k \neq j$. 
- $\bar{R}_{i j k}^{s}=0$ for arbitrary pairwise different $i, j, k$ and $s$,

- $\bar{R}_{i j k}^{i}=0$ for arbitrary $i, j, k$,

- $\bar{R}_{i j k}^{j}=R_{i j k}^{j}$ for arbitrary $j \neq i$ and $i \neq k$.

- For arbitrary $i \neq j$,

$$
\bar{R}_{i j i}^{j}=\frac{h_{j}}{h_{i}} \frac{\partial \Gamma_{i i}^{j}}{\partial x^{j}}-\frac{\partial \Gamma_{i j}^{j}}{\partial x^{i}}+\sum_{\alpha=1}^{n} \frac{h_{\alpha}}{h_{i}} \Gamma_{j \alpha}^{j} \Gamma_{i i}^{\alpha}-\frac{h_{j}}{h_{i}} \Gamma_{i i}^{j} \Gamma_{i j}^{i}-\Gamma_{i j}^{j} \Gamma_{i j}^{j}-\frac{1}{2} \frac{h_{i}^{\prime}}{h_{i}} \Gamma_{i j}^{j}+\frac{1}{2 h_{i}} h_{j}^{\prime} \Gamma_{i i}^{j},
$$

- $\bar{R}_{i j i}^{k}=\frac{h_{k}}{h_{i}} R_{i j i}^{k}$.

We again leave the proof of the lemma as an exercise (which was done many times before us, see e.g. [29, section 4]) for the reader. One needs to substitute $\Gamma_{j k}^{i}$ given by lemma 7.1 into the standard formula for the curvature

$$
R_{i j k}^{\ell}=\frac{\partial}{\partial x^{j}} \Gamma_{i k}^{\ell}-\frac{\partial}{\partial x^{k}} \Gamma_{i j}^{\ell}+\Gamma_{j s}^{\ell} \Gamma_{i k}^{s}-\Gamma_{k s}^{\ell} \Gamma_{i j}^{s} .
$$

and then carefully implement the changes in the resulting formula induced by replacing $\Gamma$ by $\bar{\Gamma}$ via formulas in lemma 7.1.

Remark 7.1. In our conventions used throughout the paper, the metric has constant curvature $K$, if (after raising the second index by $g$ ) it is given by:

$$
R_{k m}^{i j}=K\left(\delta_{k}^{i} \delta_{m}^{j}-\delta_{m}^{i} \delta_{k}^{j}\right) .
$$

Note also that because of algebraic symmetries of curvature tensors, the components listed in lemma 7.2 are essentially all components.

By our assumptions, $g$ is flat which implies that the components $\bar{R}_{i j i}^{k}$ and $\bar{R}_{i j k}^{j}$ are zero. Thus, the components of $\bar{R}$ which are 'interesting for us' (in the sense that only they have chance to be non-zero) are those considered in (44). It is convenient to raise the index $i$ by $\bar{g}$ (since $\bar{g}$ is diagonal, this operation is just the multiplication by $\frac{h_{i}}{g_{i}}$. We obtain:

$$
\begin{aligned}
\bar{R}_{i j}^{i j}= & \frac{h_{j}}{g_{i}} \frac{\partial \Gamma_{i i}^{j}}{\partial x^{j}}-\frac{h_{i}}{g_{i}} \frac{\partial \Gamma_{i j}^{j}}{\partial x^{i}}+\sum_{\alpha=1}^{n} \frac{h_{\alpha}}{g_{i}} \Gamma_{j \alpha}^{i} \Gamma_{i i}^{\alpha}-\frac{h_{j}}{g_{i}} \Gamma_{i i}^{j} \Gamma_{i j}^{i} \\
& -\frac{h_{i}}{g_{i}} \Gamma_{i j}^{j} \Gamma_{i j}^{j}-\frac{h_{i}^{\prime}}{2 g_{i}} \Gamma_{i j}^{j}+\frac{h_{j}^{\prime}}{2 g_{i}} \Gamma_{i i}^{j} .
\end{aligned}
$$

Remark 7.2. To be used in the proof of corollary 2.1, let us observe that the equation above is linear in $h_{i}$. This was expected in view of results of [29,30], and in fact the calculations above are similar to those in the corresponding places in [29]. The same is true if part of eigenvalues is complex-conjugated, see the discussion at the end of the proof.

Next, let us return to our $L$ given by (42) and employ the condition that $g L^{-k}$ are flat for $k=0, \ldots, n$ and $g L^{-n-1}$ has constant curvature $K$. Because the equation (46) are linear in $h$, and since for a polynomial $P$ the matrix $P(L)$ is diagonal $\operatorname{diag}\left(P\left(x^{1}\right), \ldots, P\left(x^{n}\right)\right)$, we have that for a polynomial $P$ (such that $P(L)$ is non-degenerate) the 'interesting components' of the curvature tensor of the metric $g P(L)^{-1}$ are given by (46) with all $h_{i}$ replaced by $P\left(x^{i}\right)$.

By our assumptions the metric $g P(L)^{-1}$ is flat for $P(t)=t^{k}$ with $k \leqslant n$ and has constant curvature $K$ for $P(t)=t^{n+1}$. Using the linearity we can combine these conditions as follows: 
for any polynomial $P=a_{n+1} t^{n+1}+\cdots+a_{0}$ (of degree $\left.\leqslant n+1\right)$ we have

$$
\begin{gathered}
\frac{P\left(x^{j}\right)}{g_{i}} \frac{\partial \Gamma_{i i}^{j}}{\partial x^{j}}-\frac{P\left(x^{i}\right)}{g_{i}} \frac{\partial \Gamma_{i j}^{j}}{\partial x^{i}}+\sum_{\alpha=1}^{n} \frac{P\left(x^{\alpha}\right)}{g_{i}} \Gamma_{j \alpha}^{j} \Gamma_{i i}^{\alpha}-\frac{P\left(x^{j}\right)}{g_{i}} \Gamma_{i i}^{j} \Gamma_{i j}^{i} \\
-\frac{P\left(x^{i}\right)}{g_{i}} \Gamma_{i j}^{j} \Gamma_{i j}^{j}-\frac{P^{\prime}\left(x^{i}\right)}{2 g_{i}} \Gamma_{i j}^{j}+\frac{P^{\prime}\left(x^{j}\right)}{2 g_{i}} \Gamma_{i i}^{j}=a_{n+1} K .
\end{gathered}
$$

Let us view (47) as an equation on the functions $\Gamma_{j k}^{i}$, the following trick allows us to find those $\Gamma_{j k}^{i}$ from this equation which are not automatically zero by lemma 7.1.

At the point $p=\left(\hat{x}_{1}, \ldots, \hat{x}_{n}\right)$ as the polynomial $P$ we take

$$
P_{0}(t)=\left(t-\hat{x}_{1}\right)\left(t-\hat{x}_{2}\right) \ldots\left(t-\hat{x}_{n}\right) \quad \text { and } \quad P_{1}(t)=t\left(t-\hat{x}_{1}\right)\left(t-\hat{x}_{2}\right) \ldots\left(t-\hat{x}_{n}\right) .
$$

For both polynomials we have $P\left(\hat{x}_{i}\right)=0$ for each $i$; for the polynomial $P_{0}$ we have

$$
P_{0}^{\prime}\left(\hat{x}_{j}\right)=\prod_{s \neq j}\left(\hat{x}_{j}-\hat{x}_{s}\right) \quad \text { and } \quad P_{0}^{\prime}\left(\hat{x}_{i}\right)=\prod_{s \neq i}\left(\hat{x}_{i}-\hat{x}_{s}\right)
$$

and for the polynomial $P_{1}$ we have

$$
P_{1}^{\prime}\left(\hat{x}_{j}\right)=\hat{x}_{j} \prod_{s \neq j}\left(\hat{x}_{j}-\hat{x}_{s}\right) \quad \text { and } \quad P_{1}^{\prime}\left(\hat{x}_{i}\right)=\hat{x}_{i} \prod_{s \neq i}\left(\hat{x}_{i}-\hat{x}_{s}\right) .
$$

Substituting $P_{0}$ into (47) and evaluating the result at $p=\left(\hat{x}_{1}, \ldots, \hat{x}_{n}\right)$ we see that most terms vanish and obtain the equation

$$
-\Gamma_{i j}^{j} \prod_{s \neq i}\left(\hat{x}_{i}-\hat{x}_{s}\right)+\Gamma_{i i}^{j} \prod_{s \neq j}\left(\hat{x}_{j}-\hat{x}_{s}\right)=0 .
$$

Similarly, doing the same with $P_{1}$, we obtain

$$
-\hat{x}_{i} \Gamma_{i j}^{j} \prod_{s \neq i}\left(\hat{x}_{i}-\hat{x}_{s}\right)+\hat{x}_{j} \Gamma_{i i}^{j} \prod_{s \neq j}\left(\hat{x}_{j}-\hat{x}_{s}\right)=2 g_{i} K .
$$

The equations (48) and (49) are two linear equations on two unknowns $\Gamma_{i j}^{j}$ and $\Gamma_{i i}^{j}$. Solving them at the point $p=\left(\hat{x}_{1}, \ldots, \hat{x}_{n}\right)$ and taking into account that this point is arbitrary, we obtain:

$$
\Gamma_{i j}^{j}=\frac{2 g_{i} K}{\left(x^{j}-x^{i}\right) \prod_{s \neq i}\left(x^{i}-x^{s}\right)} \quad \Gamma_{i i}^{j}=\frac{2 g_{i} K}{\left(x^{j}-x^{i}\right) \prod_{s \neq j}\left(x^{j}-x^{s}\right)} .
$$

Plugging them in the formulas from lemma 7.1 we obtain (at every point $\left.\left(x^{1}, \ldots, x^{n}\right)\right)$ :

$$
\begin{aligned}
& \frac{2 g_{i} K}{\left(x^{j}-x^{i}\right) \prod_{s \neq i}\left(x^{i}-x^{s}\right)}=\frac{1}{2} \frac{1}{g_{j}} \frac{\partial g_{j}}{\partial x^{i}} \\
& \frac{2 g_{i} K}{\left(x^{j}-x^{i}\right) \prod_{s \neq j}\left(x^{j}-x^{s}\right)}=-\frac{1}{2} \frac{1}{g_{j}} \frac{\partial g_{i}}{\partial x^{j}} .
\end{aligned}
$$

(Notice that swapping $i$ and $j$ in (51) we obtain the formula equivalent to (52) which was of course expected).

Remark 7.3. From (51) we see that for $K=0$ each $g_{i}$ depends on the variable $x^{i}$ only, as we claimed in remark 2.3 . In this case, the pair $(g, L)$ is not geodesically compatible. 
In what follows we assume that the dimension $n \geqslant 3$. Recall that for dimension two theorem 5 was proved in [22, theorem 10] and in [29, theorem 6.2]. See also remark 7.4 below. Take $k \notin\{i, j\}$ and consider the polynomial $P=\prod_{s \neq k}\left(t-\hat{x}_{s}\right)$ of degree $n-1$. For this polynomial, formula (47) evaluated at the point $p=\left(\hat{x}_{1}, \ldots, \hat{x}_{n}\right)$ takes the form:

$$
P\left(\hat{x}_{k}\right) \Gamma_{j k}^{j} \Gamma_{i i}^{k}-\frac{1}{2} P^{\prime}\left(\hat{x}_{i}\right) \Gamma_{i j}^{j}+\frac{1}{2} P^{\prime}\left(\hat{x}_{j}\right) \Gamma_{i i}^{j}=0 .
$$

For our $P$, we have

$$
P^{\prime}\left(\hat{x}_{i}\right)=\prod_{s \notin\{i, k\}}\left(\hat{x}_{i}-\hat{x}_{s}\right) \quad \text { and } \quad P^{\prime}\left(\hat{x}_{j}\right)=\prod_{s \notin\{j, k\}}\left(\hat{x}_{j}-\hat{x}_{s}\right) .
$$

Substituting these and (50) into (53), we obtain:

$$
\frac{4 g_{i} g_{k} K^{2}}{\left(\hat{x}_{j}-\hat{x}_{k}\right)\left(\hat{x}_{k}-\hat{x}_{i}\right) \prod_{s \neq k}\left(\hat{x}_{k}-\hat{x}_{s}\right)}-\frac{1}{2} \frac{2 g_{i} K}{\left(\hat{x}_{i}-\hat{x}_{k}\right)\left(\hat{x}_{j}-\hat{x}_{i}\right)}+\frac{1}{2} \frac{2 g_{i} K}{\left(\hat{x}_{j}-\hat{x}_{k}\right)\left(\hat{x}_{j}-\hat{x}_{i}\right)}=0 .
$$

In this equation, the factor $g_{i} K$ cancels out and we obtain the following explicit formula for $g_{k} K$ :

$$
K g_{k}\left(\hat{x}_{1}, \ldots, \hat{x}_{n}\right)=-\frac{1}{4} \prod_{s \neq k}\left(\hat{x}_{k}-\hat{x}_{s}\right) .
$$

Since this formula is fulfilled at every $p=\left(\hat{x}_{1}, \ldots, \hat{x}_{n}\right)$, it remains true if we replace all $\hat{x}_{i}$ by $x^{i}$. We see that the metric is (up to a constant factor) as in example in section 2 so $g$ and $L$ are geodesically compatible as we claimed.

Let us now comment on the case when not all eigenvalues of $L$ are real. We may again assume that all eigenvalues have algebraic multiplicity 1 . Let $k$ of them are real and the remaining $n-k$ are partitioned into complex conjugate pairs.

By [3, theorems 3.2 and 3.3], there exists a coordinate system $\left(x^{1}, \ldots, x^{k}, u^{1}, v^{1}\right.$, $\ldots, u^{\frac{n-k}{2}}, v^{\frac{n-k}{2}}$ ) such that $L$ being written in the formal coordinates

$$
\left(x^{1}, \ldots, x^{k}, z^{1}=u^{1}+i v^{1}, \bar{z}^{1}=u^{1}-i v^{1}, \ldots, \bar{z}^{\frac{n-k}{2}}=u^{\frac{n-k}{2}}-i v^{\frac{n-k}{2}}\right)
$$

takes the form

$$
L=\operatorname{diag}\left(x^{1}, \ldots, x^{k}, z^{1}, \bar{z}^{1}, \ldots, \bar{z}^{\frac{n-k}{2}}\right) .
$$

Since $L$ is $g$-symmetric, in these coordinates we also have

$$
g=\operatorname{diag}\left(g_{1}, \ldots, g_{k}, g_{k+1}, \bar{g}_{k+1}, \ldots, g_{\frac{n-k}{2}}, \bar{g}_{\frac{n-k}{2}}\right),
$$

where $g_{i}$ is a functions of $\left(x^{1}, \ldots, x^{k}, z^{1}, \ldots, \bar{z}^{\frac{n-k}{2}}\right)$ and $\bar{g}_{i}$ is its complex conjugate. Since $g$ is a usual real-valued metric, $g_{1}, \ldots, g_{k}$ are real.

Let us denote the formal coordinates $z^{1}, \ldots, \bar{z}^{\frac{n-k}{2}}$ by $x^{k+1}, \ldots, x^{n}$. Then, the Christoffel symbols and the curvature tensor are given by the same formulas as in the 'real' case, so the analogs of lemmas 7.1 and 7.2 survive and we come to the system (47). Solving the system in exactly same way as in the real case (where we used only differentiation of polynomials and algebraic manipulations, i.e., those operations that are perfectly defined over complex coordinates too) we conclude that the metric is given, up to a constant factor, by (9). Next, observe that by [8] the metric and operator of the form (9) are 
geodesically compatible even if $x^{k+1}=z^{1}, \ldots, x^{n}=\bar{z}^{\frac{n-k}{2}}$, i.e., some pairs of coordinates are complex conjugate as shown. Thus, complex roots do not affect the construction and final conclusion.

Now assume that $L$ is given by (10). Under the assumptions of theorem $5, L$ is geodesically compatible to $g$ : indeed, above we have proved that it is geodesically compatible at almost every point which implies that it is geodesically compatible at every point. Since the metric $g$ is flat, the pair $(g, L)$ is real-analytic in some coordinates (this is known and will follow from a description of geodesically compatible $(g, L)$ with $g$ flat). As we explained in section 2 , the form (9) in the coordinates $\sigma_{1}, \ldots, \sigma_{n}$ is (10). Theorem 5 is proved.

Remark 7.4. To be self-contained, we sketch a proof of theorem 5 in dimension 2. From (51) and (52) we can find the derivatives $\frac{\partial g_{1}}{\partial x^{2}}$ and $\frac{\partial g_{2}}{\partial x^{1}}$ as functions of $g_{1}, g_{2}$ :

$$
\begin{aligned}
\frac{\partial}{\partial x^{2}} g_{1} & =\frac{-4 K g_{1} g_{2}}{\left(\hat{x}_{1}-\hat{x}_{2}\right)^{2}} \\
\frac{\partial}{\partial x^{1}} g_{2} & =\frac{-4 K g_{1} g_{2}}{\left(\hat{x}_{1}-\hat{x}_{2}\right)^{2}} .
\end{aligned}
$$

Further, we assume $K=1$ without loss of generality. Plugging (55) and also (50) into (47) we obtain two linear equations in the remaining derivatives $\frac{\partial g_{1}}{\partial x^{1}}$ and $\frac{\partial g_{2}}{\partial x^{2}}$. Solving them, we obtain:

$$
\begin{aligned}
& \frac{\partial g_{1}}{\partial x^{1}}=2 \frac{g_{1}\left(\hat{x}_{1}-\hat{x}_{2}+2 g_{1}\right)}{\left(\hat{x}_{1}-\hat{x}_{2}\right)^{2}} \\
& \frac{\partial g_{2}}{\partial x^{2}}=-2 \frac{g_{2}\left(\hat{x}_{1}-2 g_{2}-\hat{x}_{2}\right)}{\left(\hat{x}_{1}-\hat{x}_{2}\right)^{2}} .
\end{aligned}
$$

The equations (55) and (56) form a PDE system of Cauchy-Frobenius type (i.e., all derivatives of unknown functions are explicitly given as algebraic expressions of unknown functions). Compatibility condition for this system is

$$
2 \frac{\left(4 g_{2}-\hat{x}_{1}+\hat{x}_{2}\right)\left(4 g_{1}+\hat{x}_{1}-\hat{x}_{2}\right)}{\left(\hat{x}_{1}-\hat{x}_{2}\right)^{4}}=0
$$

which implies that either $g_{2}=\frac{1}{4}\left(\hat{x}_{1}-\hat{x}_{2}\right)$ or $g_{1}=\frac{1}{4}\left(\hat{x}_{2}-\hat{x}_{1}\right)$. Either of these formulas combined with (55) implies (54).

\section{Acknowledgments}

We thank Jenya Ferapontov for his valuable comments and explanations. The most essential steps resulted in this paper would not have been done without outstanding research environment offered to us by Centro Internazionale per la Ricerca Matematica, Trento and the Institute of Advanced Studies, Loughborough University where the first results of the paper were obtained, and also Centre International de Rencontres Mathématiques Lumini, where the paper was finished and written. We are also grateful to Jena Universität, in particular, Ostpartnerschaft programm for supporting our research on Nijenhuis Geometry for several years. The work of Alexey Bolsinov and Andrey Konyaev was supported by Russian Science Foundation (project 17-11-01303). We thank the referees of the paper for their valuable comments and suggestions. 


\section{References}

[1] Antonowicz M and Fordy A P 1987 Coupled KdV equations with multi-Hamiltonian structures Physica D 28 345-57

[2] Arsie A and Lorenzoni P 2019 F-manifolds, multi-flat structures and Painlevé transcendents Asian J. Math. 23 877-904

[3] Bolsinov A V, Konyaev A Y and Matveev V S Nijenhuis geometry Adv. Math. (arXiv:1903.04603) (accepted)

[4] Bolsinov A V, Konyaev A Y and Matveev V S 2020 Nijenhuis geometry III: gl-regular Nijenhuis operators (arXiv:2007.09506)

[5] Bolsinov A V, Konyaev A Y and Matveev V S 2021 Applications of Nijenhuis geometry: nondegenerate singular points of Poisson-Nijenhuis structures Eur. J. Math.

[6] Bolsinov A V, Kiosak V and Matveev V S 2009 A Fubini theorem for pseudo-Riemannian geodesically equivalent metrics J. Lond. Math. Soc. 80 341-56

[7] Bolsinov A V and Matveev V S 2003 Geometrical interpretation of Benenti systems J. Geom. Phys. 44 489-506

[8] Bolsinov A and Matveev V S 2015 Local normal forms for geodesically equivalent pseudoRiemannian metrics Trans. Am. Math. Soc. 367 6719-49

[9] Brinkmann H W 1925 Einstein spaces which are mapped conformally on each other Math. Ann. 94 119-45

[10] David L and Hertling C 2017 Regular F-manifolds: initial conditions and Frobenius metrics Ann. della Scuola Norm. Super. Pisa - Cl. Sci. 17 1121-52

[11] de Vries H L 1954 Über Riemannsche Räume, die infinitesimale konforme Transformation gestatten Math. Z. $60328-47$

[12] Doyle P W 1993 Differential geometric Poisson bivectors in one space variable J. Math. Phys. 34 1314

[13] Dubrovin B A and Novikov S P 1989 Hydrodynamics of weakly deformed soliton lattices. Differential geometry and Hamiltonian theory Russ. Math. Surv. 44 29-90

[14] Eastwood M and Matveev V S 2007 Metric connections in projective differential geometry Symmetries and Overdetermined Systems of Partial Differential Equations (The IMA Volumes in Mathematics and its Applications vol 144) (New York: Springer) pp 339-51

[15] Frölicher A and Nijenhuis A 1956 Theory of vector valued differential forms. Part I Indagat. Math. $18338-50$

[16] Flood K and Gover A R 2019 Metrics in projective differential geometry: the geometry of solutions to the metrizability equation J. Geom. Anal. $292492-525$

[17] Gelfand I M and Dorfman I J 1979 Hamiltonian operators and algebraic structures associated with them Funktsional. Anal. i Prilozhen. 13 248-62 (Russian)

[18] Gover A R and Matveev V S 2017 Projectively related metrics, Weyl nullity, and metric projectively invariant equations Proc. Lond. Math. Soc. 114 242-92

[19] Ferapontov E V 2001 Compatible Poisson brackets of hydrodynamic type J. Phys. A: Math. Gen. $342377-88$

[20] Ferapontov E V and Pavlov M V 1991 Quasiclassical limit of coupled KdV equations. Riemann invariants and multi-Hamiltonian structure Physica D 52 211-9

[21] Ferapontov E V and Mokhov O I 1990 Non-local Hamiltonian operators of hydrodynamic type related to metrics of constant curvature Russ. Math. Surv. 45 218-9

[22] Ferapontov E V 1991 Hamiltonian systems of hydrodynamic type and their realization on hypersurfaces of a pseudoeuclidean space J. Sov. Math. 55 1970-95

[23] Kiosak V and Matveev V S 2009 Complete Einstein metrics are geodesically rigid Commun. Math. Phys. 289 383-400

[24] Konyaev A Y 2021 Nijenhuis geometry II: left-symmetric algebras and linearization problem for Nijenhuis operators Differ. Geom. Appl. 74101706

[25] Lancaster P and Rodman L 2005 Canonical forms for Hermitian matrix pairs under strict equivalence and congruence SIAM Rev. 47 407-43

[26] Lorenzoni P and Magri F 2005 A cohomological construction of integrable hierarchies of hydrodynamic type Int. Math. Res. Not. 2005 2087-100

[27] Matveev V S 2006 Geometric explanation of the Beltrami theorem Int. J. Geom. Methods Mod. Phys. 03 623-9 
[28] Mokhov O I 1998 Symplectic and Poisson structures on loop spaces of smooth manifolds, and integrable systems Russ. Math. Surv. 53 515-622

[29] Mokhov O I 2002 Compatible flat metrics J. Appl. Math. 2 337-70

[30] Mokhov O I 2017 Pencils of compatible metrics and integrable systems Russ. Math. Surv. 72 889-937

[31] Nijenhuis A $1951 \mathrm{X}_{n-1}$-forming sets of eigenvectors Indagat. Math. 54 200-12

[32] Pavlov M V 2003 Integrable hydrodynamic chains J. Math. Phys. 44 4134-56

[33] Pavlov M V 2018 Integrability of exceptional hydrodynamic-type systems Proc. Steklov Inst. Math. $302325-35$

[34] Sinjukov N S 1961 An invariant transformation of Riemannian spaces with common geodesics Dokl. Akad. Nauk SSSR 137 1312-4

[35] Sinjukov N S 1966 On the theory of a geodesic mapping of Riemannian spaces Dokl.Akad. Nauk SSSR 169 770-2

[36] Tsarëv S P 1991 The geometry of Hamiltonian systems of hydrodynamic type. The generalized hodograph method Math. USSR-Izv. 37 397-419

[37] Xue L and Ferapontov E V 2020 Quasilinear systems of Jordan block type and the mKP hierarchy J. Phys. A: Math. Theor. 53205202

[38] Weyl H 1956 Zur Infinitisimalgeometrie: Einordnung der projektiven und der konformen Auffasung Nachrichten von der K. Gesellschaft der Wissenschaften zu Göttingen (MathematischPhysikalische Klasse Selecta Hermann Weyl vol 1921) (Basel: Birkhäuser) 